\title{
Conformal invariance, complex structures, and the Teukolsky connection
}

\author{
Bernardo Araneda* \\ Facultad de Matemática, Astronomía y Física \\ Universidad Nacional de Córdoba \\ Instituto de Física Enrique Gaviola, CONICET \\ Ciudad Universitaria, (5000) Córdoba, Argentina
}

May 29, 2018

\begin{abstract}
We show that the Teukolsky connection, which defines generalized wave operators governing the behavior of massless fields on Einstein spacetimes of Petrov type $\mathrm{D}$, has its origin in a distinguished conformally and GHP covariant connection on the conformal structure of the spacetime. The conformal class has a (metric compatible) integrable almost-complex structure under which the Einstein space becomes a complex (Hermitian) manifold. There is a unique compatible Weyl connection for the conformal structure, and it leads to the construction of a conformally covariant GHP formalism and a generalization of it to weighted spinor/tensor fiber bundles. In particular, 'weighted Killing spinors', previously defined with respect to the Teukolsky connection, are shown to have their origin in the GHP-Weyl connection, and we show that the type D principal spinors are actually parallel with respect to it. Furthermore, we show that the existence of a conformal Killing-Yano tensor can be thought to be a consequence of the presence of a Kähler metric in the conformal class. These results provide an interpretation of the persistent hidden symmetries appearing in black hole perturbations. We also show that the preferred Weyl connection allows a natural injection of spinor fields into local twistor space and that this leads to the notion of weighted local twistors. Finally, we find conformally covariant operator identities for massless fields and the corresponding wave equations.
\end{abstract}

\section{Introduction}

Let $\left(\mathscr{M}, g_{a b}\right)$ be a 4 -dimensional spacetime with a spinor structure and Levi-Civita connection $\nabla_{a}$. Zero rest mass free fields of spin $\mathfrak{s} \in \mathbb{N}_{0} / 2$ are totally symmetric spinors

${ }^{*}$ E-mail: baraneda@famaf .unc.edu.ar 
$\varphi_{A_{1} \ldots A_{2 \mathfrak{s}}}=\varphi_{\left(A_{1} \ldots A_{2 \mathfrak{s}}\right)}$ satisfying the field equations

$$
\begin{aligned}
\nabla^{A_{1}^{\prime} A_{1}} \varphi_{A_{1} \ldots A_{2 \mathfrak{s}}} & =0, & & \text { for } \mathfrak{s}>0, \\
\square \varphi & =0, & & \text { for } \mathfrak{s}=0,
\end{aligned}
$$

where $\square=g^{a b} \nabla_{a} \nabla_{b}$. In Minkowski spacetime $\mathbb{M}=\mathbb{R}^{1,3}$, these equations have their origin in the study of the massless irreducible representations of the universal covering of the Poincaré group. In an arbitrary curved spacetime the most interesting cases of the equations correspond to spin $0,1 / 2,1$ and 2 , and our interest on them comes from studies related to the black hole stability problem. Penrose has shown in [16] that all solutions of (1.1) in $\mathbb{M}$ can be obtained from solutions of the wave equation (1.2). The process makes use of covariantly constant spinor fields, so it does not generalize to arbitrary curved spacetimes. An alternative mechanism of 'spin lowering/raising', potentially generalizable to other spacetimes, is obtained from the use of Killing spinors [15], which are totally symmetric spinor fields $\sigma^{A_{1} \ldots A_{n}}$ satisfying

$$
\nabla_{A^{\prime}}^{(A} \sigma^{\left.B_{1} \ldots B_{n}\right)}=0
$$

If $\sigma^{A_{1} \ldots A_{n}}$ is a solution to this equation, and $\varphi_{A_{1} \ldots A_{n}}$ solves (1.1), then (in Minkowski) $\Phi=\sigma^{A_{1} \ldots A_{n}} \varphi_{A_{1} \ldots A_{n}}$ solves $\square \Phi=0$. These objects are closely related to twistor theory; in particular, the 'primary spinor part' of any symmetric twistor $Z^{\alpha_{1} \ldots \alpha_{n}}$ (see section 2.6 for notation) in $\mathbb{M}$ satisfies (1.3). Killing spinors are also closely associated to the notion of 'hidden symmetries' in General Relativity, since the tensorial version of (1.3) is related to the conformal Killing-Yano equation. More precisely, Einstein spacetimes of Petrov type D have a 2-index solution $K^{A B}$ to (1.3), and this object (whose tensorial analogues are conformal Killing-Yano tensors) is responsible for the integrability of geodesic motion and the separability of the Klein-Gordon and Dirac equations on the Kerr spacetime.

In a curved spacetime, on physical grounds one would expect that, for massless fields of spin 1 and 2, it might be possible to encode the whole dynamics of the field in a complex scalar field, since it is well known that the dynamical parts of the electromagnetic and gravitational fields have only two degrees of freedom. Instead of the usual wave equation (1.2), the complex scalar field would satisfy a 'more sophisticated' wave-like equation deduced from (1.1), in which $\square$ is replaced by some normally hyperbolic operator 1 plus some potential related to the curvature. In the context of black hole perturbations, the background spacetime belongs to the type D class, so that there are two preferred null directions $o^{A}, \iota^{A}$ singled out by the geometry, and a unique non-trivial Weyl scalar $\Psi_{2}$. The GHP formalism [11] is then especially suited for this situation. The wave-like equations are known as Teukolsky, Regge-Wheeler, Fackerell-Ipser, etc.; the specific equation depends on the spin $\mathfrak{s}$ and spin weight $s$ of the variables considered, and has the general form

$$
\left(\square_{2 s}+V_{\mathfrak{s}, s}\right)\left[\Psi_{2}^{k} \Phi^{(s)}\right]=0,
$$

where $k \in \mathbb{Z} / 3, V_{\mathfrak{s}, s}$ is a complex potential, and $\Phi^{(s)}$ is the spin weight $s$ component of $\varphi_{A_{1} \ldots A_{2 \mathfrak{s}}}$ in the principal dyad. The weighted wave operator $\Phi_{p}$ acting on GHP type $\{p, 0\}$ quantities is defined as

$$
\varpi_{p}:=g^{a b} D_{a} D_{b},
$$

\footnotetext{
${ }^{1}$ i.e. a second order linear differential operator whose principal symbol is the spacetime metric.
} 
where $D_{a}:=\Theta_{a}+p B_{a}$ (for $p \in \mathbb{Z}$ ) is a modified GHP covariant derivative, with $\Theta_{a}$ the usual GHP derivative (see e.g. [11]) and $B_{a}:=-\rho n_{a}+\tau \bar{m}_{a}$ a modified GHP connection 1-form introduced in [1]. From now on we will refer to $D_{a}$ as the Teukolsky connection. The form (1.4) of the equations makes manifest their wave-like nature, which ensures the well-known properties of solutions of this kind of equations such as existence, uniqueness, and Cauchy stability in globally hyperbolic regions. Furthermore, similarly to the flat case, the field $\Psi_{2}^{k} \Phi^{(s)}$ solving (1.4) is of the form $\Psi_{2}^{k} \Phi^{(s)}=P_{s}^{A_{1} \ldots A_{2 \mathfrak{s}}} \varphi_{A_{1} \ldots A_{2 \mathfrak{s}}}$, where now $P_{s}^{A_{1} \ldots A_{2 \mathfrak{s}}}$ is given by

$$
P_{s}^{A_{1} \ldots A_{2 \mathfrak{s}}}=\Psi_{2}^{-(\mathfrak{s}-s) / 3} \iota^{\left(A_{1}\right.} \ldots \iota^{A_{\mathfrak{s}-s}} O^{A_{\mathfrak{s}-s+1}} \ldots O^{\left.A_{2 \mathfrak{s}}\right)} .
$$

Interestingly enough, it was found in [3] that (1.6) solves the equation

$$
D_{A^{\prime}}{ }^{(A} P_{s}^{\left.B_{1} \ldots B_{2 \mathfrak{s}}\right)}=0
$$

and therefore it can be considered as a Killing spinor with respect to the Teukolsky connection (in [3] these objects were called 'weighted' Killing spinors). The ordinary Killing spinor $K^{A B}=\Psi_{2}^{-1 / 3} o^{(A} \iota^{B)}$ of type D spacetimes mentioned above is just a particular case of (1.6)-(1.7). This fact suggests that there should be a deeper geometrical understanding of the Teukolsky connection, and of its relation with the 'generalized' hidden symmetries coming from (1.7), that (as far as we know) seems to be currently unknown. Since the Teukolsky equations (1.4) are central to the linear approach to the black hole stability problem (one of the major open issues in classical General Relativity), one of the main goals of this article is to provide such a geometrical understanding.

Now, the relation between (1.4) and (1.1) in the context of perturbations of a type D Einstein spacetime can be formulated as follows. Consider a family of 4-dimensional Lorentzian manifolds $\left(\mathscr{M}, g_{a b}(\varepsilon)\right)$ such that $g_{a b}(0)$ is an Einstein spacetime of Petrov type D. In order to get a second order, scalar equation from (1.1), one has to apply a first order differential operator contracting all spinor indices. Let $\mathfrak{s}=\frac{1}{2}, 1,2$ and $s=0, \pm \mathfrak{s}$. It was found in [2] that, without assuming that any field equations are satisfied, one has the operator identity

$$
P_{s}^{A_{1} \ldots A_{2 \mathfrak{s}}}\left(\nabla_{A_{1}^{\prime} A_{1}}-2 \mathfrak{s} A_{A_{1}^{\prime} A_{1}}\right) \nabla^{A_{1}^{\prime} B} \varphi_{A_{2} \ldots A_{2 \mathfrak{s}} B} \doteq\left(\square_{2 s}+V_{\mathfrak{s}, s}\right)\left[P_{s}^{A_{1} \ldots A_{2 \mathfrak{s}}} \varphi_{A_{1} \ldots A_{2 \mathfrak{s}}}\right],
$$

where "ㄹ" means equality up to linear order in $\varepsilon$ (of course this is only needed for spin $\mathfrak{s}=2$, in which the corresponding field represents the linearized Weyl curvature spinor; see [2] for details), and the 1 -form $A_{a}$ on the left is given by

$$
A_{a}=\Psi_{2}^{-1 / 3} \nabla_{a} \Psi_{2}^{1 / 3}
$$

As we want to understand the geometrical structure of (1.4) and its relation to (1.7), the identity (1.8) turns out to be appropriate for analyzing this problem since it holds independently of the field equations. From this point of view we must also understand the left hand side of (1.8) in geometrical terms, which in particular raises the question of what is the interpretation of the 1-form (1.9); namely, is it a connection form on some bundle, and if so, related to which symmetry? Moreover, a complete understanding of

\footnotetext{
${ }^{2}$ In [2] this 1-form was denoted as $-A_{a}$, and in [3] as $-\gamma_{a}$.
} 
the symmetry structures underlying (1.8) should give precise relations (geometrical or otherwise) between all objects involved in it: the spinor $P_{s}^{A_{1} \ldots A_{2 \mathfrak{s}}}$ solving (1.7), the 1-form $A_{a}$, and the Teukolsky connection $D_{a}$ and its associated wave operator $\Phi_{p}$. The aim of this work is to answer all these questions.

\subsection{Main results and overview}

In this article we prove that all the just mentioned questions can be answered by taking into account a simple observation: the field equations (1.1) are conformally invariant.

An appropriate setting for studying the present problem is then the conformal manifold $\left(\mathscr{M},\left[g_{a b}\right]\right)$ associated to our original spacetime. The analogue of the Levi-Civita connection is a Weyl connection for the conformal structure. Type D spacetimes have a naturally defined, metric compatible, almost-complex structure $J$ (which is actually integrable, thus these are complex Hermitian manifolds), and there is a unique Weyl connection compatible with it. In section 2.2 we show that (1.9) arises naturally in this way.

The (integrable) almost-complex structure is actually parallel with respect to the LeviCivita connection of a particular member of the conformal class, thus this member is a Kähler manifold. In section 2.3 we show that this implies the existence of a conformal Killing-Yano tensor (equivalently, a Killing spinor) in the original spacetime.

Furthermore, fixing a pair of null directions on the conformal manifold and using the preferred Weyl connection, in section 2.4 we construct a conformally covariant GHP formalism generalized to be valid for all spinor/tensor conformal densities, and we show that the naturally induced covariant derivative on associated vector bundles is precisely the Teukolsky connection.

The natural Weyl-GHP connection also allows us to introduce a geometrically well defined and meaningful notion of weighted Killing spinors, this gives (1.7) as a consequence of the stronger result that the canonical type D principal spinors are parallel in this more general setting (no powers of $\Psi_{2}$ needed); we prove this in section 2.5.

In section 2.6 the preferred Weyl connection is shown to give a natural injection of spinor fields into local twistor space (and a corresponding conformally invariant short exact sequence). This way we are immediately led to the consideration of weighted local twistors and to a re-derivation of the concept of weighted Killing spinors.

We then find in section 3.1 conformally covariant operator identities on spinor fields, which give the relation between field equations and conformally-GHP covariant wave equations, thus giving a version of (1.8) in a 'unified' geometrical framework.

We show the explicit relation with Teukolsky wave operators in section 3.2, and with a generalized Laplace-de Rham operator (introduced in [3]) in section 3.3.

Appendix A contains some details of the calculations left out of the main text in section 3.1.3.

\subsection{Notation and conventions}

We will work on (conformal) 4-dimensional Lorentzian spin spacetimes, with metric signature $(+---)$. Our conventions for tensor, spinor and twistor indices (and for curvature tensors, etc.) are the same as those of Penrose \& Rindler [14, 15]. We will denote as 
$\mathbb{S}_{C \ldots D^{\prime} \ldots}^{A \ldots B^{\prime} \ldots}$ the space of spinor fields (i.e. sections of spinor bundles) of the type indicated by the indices. We will furthermore use the notation $\mathbb{S}_{\{p, q\}}^{A \ldots B^{\prime} \ldots \ldots D^{\prime} \ldots}[w]$ for spinor fields with conformal weight $w$ and GHP type $\{p, q\}$.

\section{Conformal and complex structures}

\subsection{Preliminaries}

We recall some basic facts about conformal geometry, since this will be useful in the following sections. Let $\left(\mathscr{M}, g_{a b}\right)$ be a 4 -dimensional spin spacetime with spinor metric $\epsilon_{A B}$. The conformal manifold associated to this spacetime is $\left(\mathscr{M},\left[g_{a b}\right]\right)$, where $\left[g_{a b}\right]$ is the equivalence class of metrics that are conformally related to $g_{a b}$, i.e. $\widehat{g}_{a b} \in\left[g_{a b}\right]$ if and only if there exists a smooth, nowhere vanishing positive scalar function $\Omega$ such that $\widehat{g}_{a b}=\Omega^{2} g_{a b}$. The map $g_{a b} \mapsto \widehat{g}_{a b}=\Omega^{2} g_{a b}$ is called conformal transformation. For the spinor metric, this is equivalent to $\epsilon_{A B} \mapsto \widehat{\epsilon}_{A B}=\Omega \epsilon_{A B}$. For the inverse spacetime and spinor metrics, we have $\widehat{g}^{a b}=\Omega^{-2} g^{a b}$ and $\widehat{\epsilon}^{A B}=\Omega^{-1} \epsilon^{A B}$. If $\left\{o^{A}, \iota^{A}\right\}$ is a spin frame (i.e. $\epsilon_{A B}=2 o_{\left[{ }^{\iota}\right.} \iota_{B]}$ ), the spinors transform as

$$
\widehat{o}^{A}=\Omega^{w_{0}} O^{A}, \quad \widehat{\iota}^{A}=\Omega^{w_{1}} \iota^{A},
$$

for some numbers $w_{0}, w_{1} \in \mathbb{R}$ such that $w_{0}+w_{1}+1=0$. For the associated null tetrad $\left\{\ell^{a}, n^{a}, m^{a}, \bar{m}^{a}\right\}$ this is equivalent to

$$
\widehat{\ell}^{a}=\Omega^{2 w_{0}} \ell^{a}, \quad \widehat{n}^{a}=\Omega^{2 w_{1}} n^{a}, \quad \widehat{m}^{a}=\Omega^{w_{0}+w_{1}} m^{a}, \quad \widehat{\bar{m}}^{a}=\Omega^{w_{0}+w_{1}} \bar{m}^{a} .
$$

We will eventually choose particular values for $w_{0}$ and $w_{1}$.

Metrics conformally related to $g_{a b}$ can be viewed as a subbundle $\mathcal{Q} \subset T^{*} \mathscr{M} \odot T^{*} \mathscr{M}$ with fibers $\mathbb{R}^{+}$, which in turn can be understood as a principal bundle over $\mathscr{M}$ with structure group $3 \mathbb{R}^{+}$(see e.g. [5, Section 2.4]). Then one can construct vector bundles associated to $\mathcal{Q}$ known as conformally weighted line bundles, denoted by $\mathcal{E}[w]$, where $w \in \mathbb{R}$ is called conformal weight, and whose elements transform under conformal rescaling as $\widehat{\phi}=\Omega^{w} \phi$. Sections of $\mathcal{E}[w]$ are known as conformal densities of weight $w$. More generally, if $\mathbb{E}$ is a vector bundle over $\mathscr{M}$, one can construct the weighted bundle $\mathbb{E} \otimes \mathcal{E}[w]=: \mathbb{E}[w]$, the sections of which will be conformally weighted spinor/tensor fields. In particular, $\mathbb{S}_{C}^{A \ldots \ldots B^{\prime}} \ldots[w]$ will denote the space of spinor fields with conformal weight $w$.

If $\nabla_{a}$ is the Levi-Civita connection of $g_{a b}$, then under a conformal transformation, the Levi-Civita connection of the new metric $\widehat{g}_{a b}=\Omega^{2} g_{a b}$ acting on a tensor $T_{c_{1} \ldots c_{l}}^{b_{1} \ldots b_{k}}$ is

$$
\begin{aligned}
\widehat{\nabla}_{a} T_{c_{1} \ldots c_{l}}^{b_{1} \ldots b_{k}}=\nabla_{a} T_{c_{1} \ldots c_{l}}^{b_{1} \ldots b_{k}} & +K_{a}{ }^{b_{1}}{ }_{d} T_{c_{1} \ldots c_{l}}^{d \ldots b_{k}}+\ldots+K_{a}{ }^{b_{l}}{ }_{d} T_{c_{1} \ldots c_{l}}^{b_{1} \ldots d} \\
& -K_{a}{ }^{d}{ }_{c_{1}} T_{d \ldots c_{l}}^{b_{1} \ldots b_{k}}-\ldots-K_{a}{ }^{d}{ }_{c_{l}} T_{c_{1} \ldots d}^{b_{1} \ldots b_{k}},
\end{aligned}
$$

where

$$
K_{a c}{ }^{b}:=g^{b d}\left(\Upsilon_{a} g_{d c}+\Upsilon_{c} g_{d a}-\Upsilon_{d} g_{a b}\right),
$$

with $\Upsilon_{a}:=\Omega^{-1} \nabla_{a} \Omega$. For a spinor $\Psi_{C_{1} \ldots C_{l}}^{B_{1} \ldots B_{k}}$, the corresponding formula is

$$
\begin{aligned}
\widehat{\nabla}_{a} \Psi_{C_{1} \ldots C_{l}}^{B_{1} \ldots B_{k}}=\nabla_{a} \Psi_{C_{1} \ldots C_{l}}^{B_{1} \ldots B_{k}} & +\Lambda_{a D}{ }^{B_{1}} \Psi_{C_{1} \ldots C_{l}}^{D \ldots B_{k}}+\ldots+\Lambda_{a D}{ }^{B_{k}} \Psi_{C_{1} \ldots C_{l}}^{B_{1} \ldots D} \\
& -\Lambda_{a C_{1}}{ }^{D} \Psi_{D \ldots C_{l}}^{B_{1} \ldots B_{k}}-\ldots-\Lambda_{a C_{l}}{ }^{D} \Psi_{C_{1} \ldots D}^{B_{1} \ldots B_{k}},
\end{aligned}
$$

\footnotetext{
${ }^{3}$ we denote as $\mathbb{R}^{\times}$(resp. $\mathbb{R}^{+}$) the multiplicative group of real (resp. positive) numbers.
} 
where

$$
\Lambda_{a C}{ }^{B}:=\Upsilon_{A^{\prime} C} \epsilon_{A}^{B}
$$

For spinors with primed indices, the corresponding formula is deduced from (2.5) by complex conjugation, and taking into account that the 1 -form $\Upsilon_{a}$ is real. The relation between $K_{a}{ }^{b}{ }_{c}$ and $\Lambda_{a C}{ }^{B}$ is given by $K_{a}{ }^{b}{ }_{c}=\Lambda_{a C}{ }^{B} \bar{\epsilon}_{C^{\prime}}{ }^{B^{\prime}}+\bar{\Lambda}_{a C^{\prime}}{ }^{B^{\prime}} \epsilon_{C}{ }^{B}$. See [14, section 4.4].

The various parts of the curvature of $g_{a b}$ have different behaviors under conformal transformations. In particular, the Weyl spinor can be shown to be conformally invariant,

$$
\widehat{\Psi}_{A B C D}=\Psi_{A B C D}
$$

(see e.g. [15, section 6.8]), and therefore its algebraic structure is common to all metrics in the conformal class $\left[g_{a b}\right]$. This can also be seen from the conformal behavior of the GHP spin coefficients: $\kappa, \kappa^{\prime}, \sigma, \sigma^{\prime}$ are conformal densities, and by the Goldberg-Sachs theorem (valid for vacuum solutions with cosmological constant), algebraic speciality is equivalent to the existence of a shear-free null geodesic congruence:

$$
\kappa=\sigma=0
$$

(In particular, type D spaces have $\kappa=\kappa^{\prime}=\sigma=\sigma^{\prime}=0$.) The conformal behavior of the other parts of the curvature can be conveniently described by means of the Schouten tensor, which according to our conventions ([15]) is defined by

$$
P_{a b}:=-\frac{1}{2}\left(R_{a b}-\frac{R}{6} g_{a b}\right),
$$

and whose relation with the Riemann tensor is $R_{a b}{ }^{c d}=C_{a b}{ }^{c d}+4 P_{[a}{ }^{[c} g_{b]}{ }^{d]}$. Under conformal transformations, $P_{a b}$ changes as

$$
\widehat{P}_{A A^{\prime} B B^{\prime}}=P_{A A^{\prime} B B^{\prime}}-\nabla_{B B^{\prime}} \Upsilon_{A A^{\prime}}+\Upsilon_{A B^{\prime}} \Upsilon_{B A^{\prime}}
$$

\subsubsection{Weyl connections}

Below we will need the concept of a Weyl connection for the conformal structure associated to $g_{a b}$, which is a torsion-free connection $\nabla_{a}$ such that

$$
\nabla_{a} g_{b c}=-2 f_{a} g_{b c}
$$

for some 1-form $f_{a}$. Assuming $\mathbb{}_{a}$ to be fixed and calculating the conformal transformation of (2.11), we find that $f_{a}$ has the conformal behavior

$$
f_{a} \mapsto \widehat{f}_{a}=f_{a}-\Upsilon_{a}
$$

We allow $f_{a}$ to be a complex 1-form (see section 2.2). When acting on tensors, the relation between $\phi_{a}$ and the Levi-Civita connection is given by a formula analogous to (2.3), but replacing $K_{a}{ }^{b}{ }_{c}$ with the tensor

$$
Q_{a}{ }^{b}{ }_{c}:=g^{b d}\left(f_{a} g_{d c}+f_{c} g_{d a}-f_{d} g_{a b}\right) .
$$

The action of $\not_{a}$ on spinors is analogous to (2.5) and its complex conjugate, but replacing $\Lambda_{a C}{ }^{B}$ and $\bar{\Lambda}_{a C}{ }^{B}$ respectively for

$$
W_{a C}{ }^{B}=f_{A^{\prime} C} \epsilon_{A}^{B}, \quad \widetilde{W}_{a C^{\prime}}{ }^{B^{\prime}}=f_{A C^{\prime}} \bar{\epsilon}_{A^{\prime}}{ }^{B^{\prime}} .
$$


We must use the object $\widetilde{W}_{a C^{\prime}} B^{\prime}$ instead of $\bar{W}_{a C^{\prime}} B^{\prime}$ because the 1-form $f_{a}$ can be complex, i.e. $\bar{f}_{a} \neq f_{a}$ (note that in the case $f_{a}$ real, we have $\widetilde{W}_{a C^{\prime}}{ }^{\prime}=\bar{W}_{a C^{\prime}}{ }^{B^{\prime}}$ ).

If $\Psi$ is an arbitrary spinor/tensor field with conformal weight $w \neq 0$ and $\nabla_{a}$ is a Weyl connection, then $\bigotimes_{a} \Psi$ is not a conformal density. A covariant derivative operator that maps conformal densities of weight $w$, to conformal densities (of the same weight), can be constructed as

$$
\mathscr{C}_{a} \Psi=\not_{a} \Psi+w f_{a} \Psi
$$

It then follows that $\widehat{\mathscr{C}_{a} \Psi}=\Omega^{w} \mathscr{C}_{a} \Psi$. We say that $\mathscr{C}_{a}$ is a conformally covariant derivative. More generally, we will say that a linear differential operator $P$ is conformally covariant if, when acting on conformal spinor/tensor densities $\Psi$ of weight $w$, it satisfies $\widehat{P(\Psi)}=$ $\Omega^{w^{\prime}} P(\Psi)$ for some $w^{\prime} \in \mathbb{R}$.

\section{$2.2 \quad$ Almost-complex structure}

Consider the conformal manifold $\left(\mathscr{M},\left[g_{a b}\right]\right)$ associated to $g_{a b}$ (whose Levi-Civita connection is $\nabla_{a}$ ). Suppose that there exists an almost-complex structure $J$ : a linear map $J: T \mathscr{M} \rightarrow T \mathscr{M}$ such that $J^{2}=-$ Id (the identity map on $T \mathscr{M}$ ). Assume also that $J$ is metric compatible, i.e. $g(J X, J Y)=g(X, Y)$ for all $X, Y \in T \mathscr{M}$. In index notation, $J_{a}{ }^{b}$ is a compatible almost-complex structure if $J_{a}^{c} J_{c}^{b}=-\delta_{a}^{b}$ and $J_{a}^{c} J_{b}{ }^{d} g_{c d}=g_{a b}$. (Note that these conditions imply $J_{(a b)}=0$.) The resulting triple $\left(\mathscr{M},\left[g_{a b}\right], J\right)$ is called a conformal almost-Hermitian manifold. As discussed in [4] (see also e.g. [12]), there is a unique Weyl connection $\not_{a}$ on $\left(\mathscr{M},\left[g_{a b}\right], J\right)$ that is compatible with $J$, i.e. such that

$$
\not_{b} J_{a}{ }^{b}=0
$$

Acting on an arbitrary tensor field, such a connection is given by a formula analogous to (2.3) but replacing $K_{a}{ }^{b}{ }_{c}$ with (2.13), where the 1 -form $f_{a}$ is canonically found to be

$$
f_{a}=-\frac{1}{2} J_{b}{ }^{c} \nabla_{c} J_{a}^{b} .
$$

Now, for any choice of $g_{a b} \in\left[g_{a b}\right]$, a spin frame $\left\{o^{A}, \iota^{A}\right\}, o_{A} \iota^{A}=1$, or equivalently the associated null tetrad $\left\{N_{\mathbf{a}}^{a}\right\}=\left\{\ell^{a}, n^{a}, m^{a}, \bar{m}^{a}\right\}(\mathbf{a}=0, \ldots, 3)$, always defines an almostcomplex structure a: 4

$$
J_{a}^{b}=i\left(\ell_{a} n^{b}-n_{a} \ell^{b}+\bar{m}_{a} m^{b}-m_{a} \bar{m}^{b}\right)
$$

As far as we know, this structure was first found in 77; then rediscovered in [4] (see also [8] and [13, II.2.10]). In spinor form, (2.18) is $J_{a}^{b}=i\left(o_{A} \iota^{B}+\iota_{A} o^{B}\right) \bar{\epsilon}_{A^{\prime}}{ }^{B^{\prime}}$. Although (2.18) is well defined in any (4-dimensional) spacetime, it is particularly significant for the case of Petrov type $\mathrm{D}$, since then we have a pair of null directions $O^{A}, \iota^{A}$ determined by the geometry and $J_{a}{ }^{b}$ is naturally associated to this structure.

\footnotetext{
${ }^{4}$ note that (2.18) is a complex-valued tensor. For this reason, in 8] it is referred to as a 'modified' almost-complex structure. A real almost-complex structure can be constructed as $J_{a}^{b}=-\ell_{a} \ell^{b}+n_{a} n^{b}-$ $i m_{a} \bar{m}^{b}+i \bar{m}_{a} m^{b}$, but this has a number of undesirable properties that make it unsuitable for our purposes, see [8, Chapter VIII].
} 
Remark 2.1 (Chirality). Note that we are choosing the spinors $O^{A}, \iota^{A}$ instead of $\bar{o}^{A^{\prime}}, \bar{\iota}^{A^{\prime}}$; the latter choice would give the equally valid almost-complex structure $\tilde{J}_{a}^{b}=i\left(\bar{o}_{A^{\prime}} \bar{l}^{B^{\prime}}+\right.$ $\left.\bar{\iota}_{A^{\prime}} O^{B^{\prime}}\right) \epsilon_{A}{ }^{B}$, and the corresponding 1-form (2.17) would now be $\bar{f}_{a}$. In this sense we can consider our treatment as chiral, since left-and right-handed spinors are not treated on an equal footing. This is because we want to study the left-handed fields (1.1).

Note that (2.18) does not depend on the representatives of the conformal and GHP classes (i.e. it is invariant under conformal and GHP transformations). Note also that $J_{a}{ }^{b}$ is compatible with the conformal structure: for any $g_{a b} \in\left[g_{a b}\right]$, we have $J_{a}{ }^{c} J_{b}{ }^{d} g_{c d}=g_{a b}$, therefore a type D spacetime is a conformal almost-Hermitian manifold with almostcomplex structure (2.18). The associated (unique) compatible Weyl connection (2.17) is calculated to be

$$
f_{a}=\rho n_{a}+\rho^{\prime} \ell_{a}-\tau \bar{m}_{a}-\tau^{\prime} m_{a} .
$$

Under conformal rescalings, we have of course $f_{a} \mapsto \widehat{f}_{a}=f_{a}-\Upsilon_{a}$.

Remark 2.2 (The 1-form (1.9)). In an Einstein spacetime of Petrov type D, the Bianchi identities in GHP form are $\mathrm{b} \Psi_{2}=3 \rho \Psi_{2}$ and $\partial \Psi_{2}=3 \tau \Psi_{2}$ (together with the primed versions), and they imply that the 1-form (2.19) coincides exactly with (1.9), i.e. $f_{a} \equiv \Psi_{2}^{-1 / 3} \nabla_{a} \Psi_{2}^{1 / 3} \equiv A_{a}$. It is important to emphasize, however, that the Bianchi identity $\nabla^{A^{\prime} A} \Psi_{A B C D}=0$ is not conformally invariant; therefore by writing (2.19) in this form one is explicitly breaking conformal covariance.

\subsection{Kähler structure}

An almost-complex manifold is actually a complex manifold if the almost-complex structure is integrable. By the Newlander-Nirenberg theorem, the almost-complex structure is integrable if and only if the associated Nijenhuis tensor ${ }^{J} N(X, Y)$ vanishes for all $X, Y \in T \mathscr{M}$, where ${ }^{J} N(X, Y) \equiv[X, Y]+J[J X, Y]+J[X, J Y]-[J X, J Y]$ and $[\cdot, \cdot \cdot]$ is the Lie bracket of vector fields. In index notation, this is ${ }^{J} N_{b c}{ }^{a}=-J_{b}{ }^{d} \partial_{d} J_{c}{ }^{a}+J_{c}{ }^{d} \partial_{d} J_{b}{ }^{a}+$ $J_{d}{ }^{a}\left(\partial_{b} J_{c}{ }^{d}-\partial_{c} J_{b}{ }^{d}\right)$. Note that, in this expression, $\partial_{a}$ can be replaced by any torsion-free connection; in particular, using the Weyl connection $\nabla_{a}$ applied to (2.18) we find

$$
\not_{a} J_{b}{ }^{c}=2 i \bar{\epsilon}_{B^{\prime}} C^{\prime}\left[\left(-\kappa n_{a}+\sigma \bar{m}_{a}\right) \iota_{B} \iota^{C}+\left(-\kappa^{\prime} \ell_{a}+\sigma^{\prime} m_{a}\right) o_{B} O^{C}\right] .
$$

The Nijenhuis tensor then vanishes for a type D spacetime (see (2.8)), thus the almostcomplex structure is integrable and the space can be regarded as a complex manifold. Complex coordinates are obtained by integrating linear combinations of the type $(1,0)$ and $(0,1)$ forms with respect to the decomposition $5\left(T^{*} \mathscr{M}\right)^{\mathbb{C}}=T^{*} \mathscr{M}^{+} \oplus T^{*} \mathscr{M}^{-}$induced by $J: T^{*} \mathscr{M}^{+}$is spanned by $\left\{\ell_{a} d x^{a}, m_{a} d x^{a}\right\}$, and $T^{*} \mathscr{M}^{-}$by $\left\{n_{a} d x^{a}, \bar{m}_{a} d x^{a}\right\}$. Now, since $J$ is compatible with $g_{a b}$, we actually get a Hermitian manifold. The 2 -form defined by $\omega(X, Y):=g(J X, Y)$, namely $\omega_{a b}=J_{a b}$, is called Kähler form. A Kähler manifold is a Hermitian manifold $\left(\mathscr{M}, g_{a b}\right)$ for which the Kähler form is closed, and it is a theorem that $\omega$ is closed if and only if the almost-complex structure $J$ is parallel with respect to

\footnotetext{
${ }^{5}$ this decomposition is valid because the complex tensor $J_{a}{ }^{b}$ has eigenvalues $+i,+i,-i,-i$; for this reason one does not consider $i \delta_{a}{ }^{b}$ as an almost-complex structure, since it has eigenvalues $+i,+i,+i,+i$ (and because it is not metric compatible).
} 
the Levi-Civita connection of $g_{a b}$. It can be checked that in general $J$ is not parallel with respect to $\nabla_{a}$; but from (2.20) we know that, for type D spacetimes, $J$ is parallel with respect to $\nabla_{a}$. Now, it is well known that a Weyl connection is actually the Levi-Civita connection of some $g_{a b} \in\left[g_{a b}\right]$ if the 1 -form $f_{a}$ is closed. For Einstein type D spaces, by Remark 2.2 we know that $f_{a}=\nabla_{a} \log \Psi_{2}^{1 / 3}$ and hence it is closed, thus $\mathbb{}_{a}$ is the Levi-Civita connection of $\phi_{a b}:=\Psi_{2}^{2 / 3} g_{a b}$. Therefore, $\left(\mathscr{M}, \phi_{a b}\right)$ is a Kähler manifold, i.e. Einstein spacetimes of Petrov type $D$ are conformal (with generally complex conformal factor) to Kähler spaces (see also [8]).

We will now show that the existence of a Kähler metric in the conformal structure is directly related to the hidden symmetries associated to conformal Killing-Yano tensors and Killing spinors. A conformal Killing-Yano tensor is a 2-form $Z_{a b}$ satisfying the equation

$$
\nabla_{(a} Z_{b) c}=g_{a b} \xi_{c}-g_{c(a} \xi_{b)},
$$

where (in dimension $d$ ) $\xi_{a}=-\frac{1}{(d-1)} \nabla_{b} Z_{a}{ }^{b}$. We have:

Lemma 2.3. Let $\left(\mathscr{M},\left[g_{a b}\right], J\right)$ be a d-dimensional conformal Hermitian manifold, and assume that there exists a Kähler metric $\widetilde{g}_{a b}$ in the conformal class. Then the metric $g_{a b}=\Omega^{-2} \widetilde{g}_{a b}$ admits a conformal Killing-Yano tensor, given by $Z_{a b}=\Omega^{-1} J_{a b}$, where $J_{a b}:=g_{b c} J_{a}^{c}$.

Proof. Let $\widetilde{\nabla}_{a}$ be the Levi-Civita connection of the Kähler metric $\widetilde{g}_{a b}$, and let $\nabla_{a}$ be the corresponding one to the conformally related metric $g_{a b}=\Omega^{-2} \widetilde{g}_{a b}$. In what follows, raising and lowering of indices is performed with $g_{a b}$ and its inverse $g^{a b}$. By hypothesis we have $\widetilde{\nabla}_{a} J_{b}{ }^{c}=0$; therefore symmetrizing and using the relation (2.3) , we get

$$
\widetilde{\nabla}_{(a} J_{b)}{ }^{c}=0=\Omega \nabla_{(a}\left[\Omega^{-1} J_{b)}{ }^{c}\right]+g_{a b} \Upsilon^{d} J_{d}^{c}+g_{(a}{ }^{c} J_{b)}{ }^{d} \Upsilon_{d}
$$

Using that $\Omega \nabla_{a}\left[\Omega^{-1} J_{b}{ }^{a}\right]=-(d-1) J_{b}{ }^{a} \Upsilon_{a}$ and defining $Z_{a}{ }^{b}=\Omega^{-1} J_{a}{ }^{b}$, it is straightforward to show that the previous equation is equivalent to

$$
\nabla_{(a} Z_{b)}{ }^{c}=-\frac{1}{(d-1)} g_{a b} \nabla_{d} Z^{c d}+\frac{1}{(d-1)} g_{(a}^{c} \nabla_{|d|} Z_{b)}{ }^{d},
$$

thus $Z_{a b}$ is a conformal Killing-Yano tensor.

In 4 dimensions, the 2-form $Z_{a b}$ in Lemma 2.3 can be written in spinor language as $Z_{a b}=\psi_{A B} \bar{\epsilon}_{A^{\prime} B^{\prime}}+\chi_{A^{\prime} B^{\prime}} \epsilon_{A B}$ for some symmetric spinors $\psi_{A B}$ and $\chi_{A^{\prime} B^{\prime}}$, and the conformal Killing-Yano equation (2.21) implies that $\psi_{A B}$ and $\chi_{A^{\prime} B^{\prime}}$ are Killing spinors. Thus, the existence of these objects can be thought to be a consequence of the presence of a Kähler metric in the conformal class of the spacetime.

\subsection{Conformally covariant GHP formalism}

Consider a fixed spacetime $\left(\mathscr{M}, g_{a b}\right)$. The GHP formalism is a framework suited for spacetimes in which two null directions can be tied to the geometry. One adapts a null frame $\left\{N_{\mathbf{a}}^{a}\right\}=\left\{\ell^{a}, n^{a}, m^{a}, \bar{m}^{a}\right\}$ to the structure of null directions, in such a way that the remaining freedom for selecting a frame is reduced from $\mathrm{SO}(1,3)^{\uparrow}$ to a 2-dimensional subgroup given by $\mathbb{R}^{\times} \times \mathrm{U}(1)\left(\cong \mathbb{C}^{\times}\right)$, whose action on a null tetrad is

$$
\ell^{a} \rightarrow a \ell^{a}, \quad n^{a} \rightarrow a^{-1} n^{a}, \quad m^{a} \rightarrow z m^{a}, \quad \bar{m}^{a} \rightarrow \bar{z} \bar{m}^{a},
$$


for $a \in \mathbb{R}^{\times}$and $z \in \mathrm{U}(1)$. Spinors, tensors, equations, etc. are projected on the null frame, so that the components $\eta$ of an arbitrary spinor/tensor field get changed under a GHP transformation (2.24), in the form $\eta \mapsto \lambda^{p} \bar{\lambda}^{q} \eta$ for some $p, q \in \mathbb{Z}$ (where $\lambda=(a z)^{1 / 2}$ ); i.e. they form representations of the GHP group. These quantities are known as GHP weighted quantities of type $\{p, q\}$, and can be thought of as sections of vector bundles $\mathbb{E}_{\{p, q\}}$ associated to the GHP representations. The covariant derivative on $\mathbb{E}_{\{p, q\}}$ is

$$
\Theta_{a}=\partial_{a}+p \omega_{a}+q \bar{\omega}_{a}
$$

where $\omega_{a}$ is the GHP connection

$$
\omega_{a}=-\epsilon n_{a}+\epsilon^{\prime} \ell_{a}-\beta^{\prime} m_{a}+\beta \bar{m}_{a} .
$$

Consider now the conformal manifold $\left(\mathscr{M},\left[g_{a b}\right]\right)$, which is a structure weaker than the spacetime. We wish to use the preferred Weyl connection (2.19) to construct a conformally covariant GHP formalism. Let $\pi_{P}: P \rightarrow \mathscr{M}$ be a principal fiber bundle over the conformal manifold, whose typical fiber over $x \in \mathscr{M}$ is the set of basis $\left\{N_{\text {a }}^{a}\right\}$ of $T_{x} \mathscr{M}$ such that for any $\widehat{g}_{a b} \in\left[g_{a b}\right]$, there exists $\alpha>0$ such that $\widehat{g}_{a b} N_{\mathbf{a}}^{a} N_{\mathbf{b}}^{b}=\alpha^{2} \nu_{\mathbf{a b}}$, where $\nu_{\mathbf{a b}}$ is defined by $\nu_{01}=1=-\nu_{23}$, with all other components being zero. The structure group of $P$ is $G=\mathrm{SO}(1,3)^{\uparrow} \times \mathbb{R}^{+}$(see e.g. [9]). The Weyl connection $\mathbb{}_{a}$ on $\left(\mathscr{M},\left[g_{a b}\right]\right)$ allows to define a notion of parallel transport on $\mathscr{M}$, which in turn induces a (local) connection 1-form on $P$ given by $\psi_{a}{ }^{\mathbf{b}} \mathbf{c}=N_{b}^{\mathbf{b}} \not_{a} N_{\mathbf{c}}^{b}$ (where $N_{a}^{\mathbf{a}}$ is the frame dual to $N_{\mathbf{a}}^{a}$ ). Note that, since (2.19) is generally complex, $\psi_{a}{ }_{\mathbf{b}} \mathbf{c}$ will take values in the complexification of the Lie algebra $\mathfrak{g}=\operatorname{Lie}(G)$, namely $\psi \in T^{*} \mathscr{M} \otimes \mathfrak{g}^{\mathbb{C}}$, with $\mathfrak{g}^{\mathbb{C}}:=\mathfrak{g} \otimes \mathbb{C}$. Now, in the GHP formalism one fixes a pair of null directions $\ell^{a}, n^{a}$. The subgroup of $G$ that preserves these null directions in the conformal structure is $G_{o}=\mathbb{R}^{\times} \times \mathrm{U}(1) \times \mathbb{R}^{+}$, whose action on the frame $\left\{N_{\mathbf{a}}^{a}\right\}$ is now

$$
\ell^{a} \rightarrow \Omega^{2 w_{0}} a \ell^{a}, \quad n^{a} \rightarrow \Omega^{2 w_{1}} a^{-1} n^{a}, \quad m^{a} \rightarrow \Omega^{w_{0}+w_{1}} z m^{a}, \quad \bar{m}^{a} \rightarrow \Omega^{w_{0}+w_{1}} \bar{z} \bar{m}^{a},
$$

where $a \in \mathbb{R}^{\times}, z \in \mathrm{U}(1), \Omega \in \mathbb{R}^{+}$, and $w_{0}, w_{1}$ are two real numbers that satisfy $w_{0}+w_{1}+$ $1=0$ (in accordance to (2.1) ). The reduction $G \rightarrow G_{o}$ gives in turn a reduction of $P$ to another principal bundle $B$ with structure group $G_{o}$, and the induced connection form on $B$ is obtained as the parts of $\psi_{a} \mathbf{b}_{\mathbf{c}}$ that do not transform covariantly under $G_{o}$. It is straightforward to check that these parts are $\psi_{a}{ }_{0}, \psi_{a}{ }^{1}, \psi_{a}{ }^{2}$ and $\psi_{a}{ }^{3}{ }_{3}$, which transform as

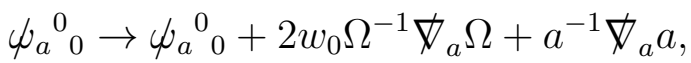

$$
\begin{aligned}
& \psi_{a}{ }^{1}{ }_{1} \rightarrow \psi_{a}{ }^{1}{ }_{1}+2 w_{1} \Omega^{-1} \nabla_{a} \Omega+a \not_{a} a^{-1}, \\
& \psi_{a}^{2}{ }_{2} \rightarrow \psi_{a}^{2}{ }_{2}+\Omega^{-1} \nabla_{a} \Omega+z^{-1} \nabla_{a} z \text {, } \\
& \psi_{a}{ }^{3}{ }_{3} \rightarrow \psi_{a}{ }^{3}{ }_{3}+\Omega^{-1} \nabla_{a} \Omega+z \nabla_{a} z^{-1} \text {. }
\end{aligned}
$$

By looking at these transformation behaviors, we can isolate the parts that transform only under each separate subgroup in the product $G_{o}=\mathbb{R}^{\times} \times \mathrm{U}(1) \times \mathbb{R}^{+}$; these are $w_{1} \psi_{a}{ }_{0}{ }_{0}-w_{0} \psi_{a}{ }^{1}{ }_{1}=: K_{a}$ for $\mathbb{R}^{\times}, \frac{1}{2}\left(\psi_{a}{ }^{3}{ }_{3}-\psi_{a}{ }^{2}{ }_{2}\right)=: L_{a}$ for $\mathrm{U}(1)$, and $\frac{1}{2}\left(\psi_{a}{ }^{2}{ }_{2}+\psi_{a}{ }^{3}{ }_{3}\right)=f_{a}$ for $\mathbb{R}^{+}$, since we have

$$
K_{a} \rightarrow K_{a}+a \not_{a} a^{-1}, \quad L_{a} \rightarrow L_{a}+z \not_{a} z^{-1}, \quad f_{a} \rightarrow f_{a}+\Omega \not_{a} \Omega^{-1} .
$$


The connection form on $B$ is then $\psi_{a}=\left(K_{a}, L_{a}, f_{a}\right)$, which takes values in the complexified Lie algebra $\mathfrak{g}_{o}^{\mathbb{C}}=(\mathbb{R} \oplus \mathfrak{u}(1) \oplus \mathbb{R}) \otimes \mathbb{C} \cong \mathbb{C} \oplus \mathbb{C} \oplus \mathbb{C}$.

Consider now a conformal tensor density, and project it over the frame $\left\{N_{\mathrm{a}}^{a}\right\}$ and its dual. These components (denoted generically by $\eta$ ) will be appropriately rescaled under (2.27); i.e., they form representations $\Pi_{b, s, w}: G_{o} \rightarrow \mathrm{GL}(\mathbb{C})$ of $G_{o}$ given by

$$
\Pi_{b, s, w}(a, z, \Omega) \eta:=a^{b} z^{s} \Omega^{w} \eta, \quad \eta \in \mathbb{C}, \quad(a, z, \Omega) \in G_{o}
$$

where $b$ and $s$ are integer numbers and $w \in \mathbb{R}$. The components $\eta$ are then said to have boost weight $b$, spin weight $s$, and conformal weight $w$. In the spinor approach it is more natural to use instead the alternative $\{p, q\}$ weights defined by $b=(p+q) / 2, s=(p-q) / 2$, in which case we can denote the representation (2.33) as $\Pi_{p, q, w}$. These quantities will be said to have type $\{w ; p, q\}$. The associated representation of the Lie algebra is

$$
\pi_{p, q, w}(x, y, v) \eta=\left(p \frac{(x+y)}{2}+q \frac{(x-y)}{2}+w v\right) \eta .
$$

For (conformal) tensor fields, the components are sections of the associated vector bundles

$$
\mathbb{E}_{\{p, q\}}[w]:=B \times_{\Pi_{p, q, w}} \mathbb{C} .
$$

A covariant derivative on this structure can be induced by the connection form $\psi_{a}$ on $B$ :

Lemma 2.4. The covariant derivative on the associated vector bundles $\mathbb{E}_{\{p, q\}}[w]$ is

$$
\oplus_{a} \eta=\partial_{a} \eta+\pi_{p, q, w}\left(\psi_{a}\right) \eta
$$

Explicitly, this is

$$
\oplus_{a} \eta=\partial_{a} \eta+w f_{a} \eta+p\left(\omega_{a}+B_{a}\right) \eta+q\left(\bar{\omega}_{a}+C_{a}\right) \eta
$$

where $\omega_{a}$ is the GHP connection form, and the complex 1-forms $B_{a}, C_{a}$ are defined by

$$
\begin{aligned}
& B_{a}:=\frac{1}{2}\left(K_{a}+L_{a}\right)=w_{1}\left(\rho n_{a}-\tau \bar{m}_{a}\right)-w_{0}\left(\rho^{\prime} \ell_{a}-\tau^{\prime} m_{a}\right), \\
& C_{a}:=\frac{1}{2}\left(K_{a}-L_{a}\right)=w_{1}\left(\rho n_{a}-\tau^{\prime} m_{a}\right)-w_{0}\left(\rho^{\prime} \ell_{a}-\tau \bar{m}_{a}\right) .
\end{aligned}
$$

Proof. This is deduced from our discussion above, and from the general formula for the covariant derivative induced on associated vector bundles.

Remark 2.5 (The Teukolsky connection). Choose the conformal weights $w_{0}=0$ and $w_{1}=-1$ in (2.1). For quantities with $w=0$ and $q=0$, $\oplus_{a}$ coincides exactly with the Teukolsky derivative $D_{a}$. (Note that, since the fields we are interested in -namely $\varphi_{A_{1} \ldots A_{25}-}$ have no primed indices, no q-weight will appear in our general formulae.) For conformal weight $w \neq 0$, by writing $f_{a}$ in the form (1.9) we see that the covariant derivative (2.37) describes exactly the combination of Teukolsky derivatives and different powers of $\Psi_{2}^{1 / 3}$ that appear in formulae involving $\iota^{A}$, see section 3.2 .

From the discussion above we can conclude:

Theorem 2.6. The Teukolsky connection is originated in the covariant derivative naturally induced on the vector bundles (2.35) associated to representations of the GHPconformal symmetry. 
An important property of (2.36) is the following:

Proposition 2.7. $\oplus_{a}$ commutes with the GHP prime operation]:

$$
\left(\oplus_{a} \eta\right)^{\prime}=\bigoplus_{a} \eta^{\prime}
$$

Proof. If $\eta$ is of type $\{w ; p, q\}$, then $\eta^{\prime}$ is of type $\left\{w^{\prime} ;-p,-q\right\}$, where $w^{\prime}$ is given by $w^{\prime}=w-\left(w_{0}-w_{1}\right)(p+q)$. Using that

$$
\begin{aligned}
& B_{a}^{\prime}=-\left(w_{0}-w_{1}\right) f_{a}-B_{a}, \\
& C_{a}^{\prime}=-\left(w_{0}-w_{1}\right) f_{a}-C_{a},
\end{aligned}
$$

the result follows easily by calculating both sides of (2.40).

As in the usual GHP formalism, this result allows one to halve the number of calculations. On the other hand, $\oplus_{a}$ does not commute with complex conjugation. This is related to our chiral treatment, see Remark 2.1.

For spinor/tensor fields that are GHP and conformally weighted and have a nontrivial structure of indices, we must complement (2.37) with the objects (2.13) and (2.14) in order to get a derivative operator that is covariant under both kinds of transformations. To this end, we define a covariant derivative $\mathscr{C}_{a}$ that acts on a vector $v^{b}$ and a spinor $\kappa^{B}$, both with conformal weight $w$ and GHP type $\{p, q\}$, as

$$
\begin{aligned}
& \mathscr{C}_{a} v^{b}=\nabla_{a} v^{b}+\left[w f_{a}+p\left(\omega_{a}+B_{a}\right)+q\left(\bar{\omega}_{a}+C_{a}\right)\right] v^{b}, \\
& \mathscr{C}_{a} \kappa^{B}=\nabla_{a} \kappa^{B}+\left[w f_{a}+p\left(\omega_{a}+B_{a}\right)+q\left(\bar{\omega}_{a}+C_{a}\right)\right] \kappa^{B} .
\end{aligned}
$$

The extension of the action of $\mathscr{C}_{a}$ to a spinor/tensor field with an arbitrary structure of indices follows straightforwardly. One can then check that, under conformal and GHP transformations, for an arbitrary spinor/tensor field $\Psi$ of type $\{w ; p, q\}$ we have

$$
\mathscr{C}_{a} \Psi \rightarrow \Omega^{w} \lambda^{p} \bar{\lambda}^{q} \mathscr{C}_{a} \Psi
$$

Finally, the projection of $\mathscr{C}_{a}$ on a null tetrad defines the conformally weighted GHP operators

$$
\mathrm{p}_{\mathscr{C}}:=\ell^{a} \mathscr{C}_{a}, \quad \mathrm{p}_{\mathscr{C}}^{\prime}:=n^{a} \mathscr{C}_{a}, \quad \partial_{\mathscr{C}}:=m^{a} \mathscr{C}_{a}, \quad \chi_{\mathscr{C}}^{\prime}:=\bar{m}^{a} \mathscr{C}_{a}
$$

We emphasize that, with the above definition of $\mathscr{C}_{a}$, these operators act on arbitrary spinor/tensor conformal densities. For the particular case of a scalar conformal density $\eta$ of type $\{w ; p, q\}$, we have

$$
\begin{aligned}
& \mathrm{p}_{\mathscr{C}} \eta=\left[\mathrm{p}+\left(w+(p+q) w_{1}\right) \rho\right] \eta, \\
& \mathrm{b}_{\mathscr{C}}^{\prime} \eta=\left[\mathrm{p}^{\prime}+\left(w-(p+q) w_{0}\right) \rho^{\prime}\right] \eta, \\
& \partial_{\mathscr{C}} \eta=\left[\partial+\left(w+p w_{1}-q w_{0}\right) \tau\right] \eta, \\
& \partial_{\mathscr{C}}^{\prime} \eta=\left[\partial^{\prime}+\left(w-p w_{0}+q w_{1}\right) \tau^{\prime}\right] \eta,
\end{aligned}
$$

this way we recover the operators defined by Penrose \& Rindler in [14, section 5.6] (see Eqs. (5.6.36) in that reference).

\footnotetext{
${ }^{6}$ We recall that the GHP prime operation is defined as the transformation $o^{A} \rightarrow i \iota^{A}, \iota^{A} \rightarrow i o^{A}$.
} 


\subsection{Parallel and Killing spinors}

As we have seen, the almost-comlpex structure (2.18) of type D spacetimes determines a unique Weyl connection on the conformal structure, and thus we have a naturally induced covariant derivative on weighted spinor/tensor bundles, given by (2.44), (2.43), (2.36). This leads us to a geometrically meaningful generalization of some interesting differential equations; for example, we are naturally led to the notion of a weighted Killing spinor as an element $\omega^{A_{1} \ldots A_{n}}$ of $\mathbb{S}_{\{p, q\}}^{A_{1} \ldots A_{n}}[w]$ that satisfies the equation

$$
\mathscr{C}_{A^{\prime}}\left(A^{\left.B_{1} \ldots B_{n}\right)}=0\right.
$$

A stronger condition is that of a parallel spinor, i.e. a solution of

$$
\mathscr{C}_{a} \omega^{B_{1} \ldots B_{n}}=0
$$

Lemma 2.8. Let $\left(\mathscr{M},\left[g_{a b}\right], J\right)$ be the conformal almost-Hermitian manifold associated to an Einstein spacetime $\left(\mathscr{M}, g_{a b}\right)$, where $J$ is given by (2.18) for an arbitrarily chosen pair of null directions $\left\{o^{A}, \iota^{A}\right\}$, with $o^{A} \in \mathbb{S}_{\{1,0\}}^{A}\left[w_{0}\right], \iota^{A} \in \mathbb{S}_{\{-1,0\}}^{A}\left[w_{1}\right]$ and $o_{A} \iota^{A}=1$. Then the following two are equivalent:

(i) The spacetime is algebraically special, with principal null direction $o^{A}$.

(ii) The spinor $o^{A}$ is parallel with respect to the naturally induced covariant derivative, namely $\mathscr{C}_{a} O^{B}=0$.

In particular, the spacetime is of Petrov type $D$ if and only if both spinors are parallel, $\mathscr{C}_{a} o^{B}=0=\mathscr{C}_{a} \iota^{B}$.

Proof. A straightforward calculation shows that (for any conformal weights $w_{0}, w_{1}$ in (2.1) )

$$
\mathscr{C}_{a} O^{B}=\left(-\kappa n_{a}+\sigma \bar{m}_{a}\right) \iota^{B} .
$$

By the Goldberg-Sachs theorem, algebraic speciality is equivalent to $\kappa=\sigma=0$, thus the result $(i) \Leftrightarrow(i i)$ follows. Since $\mathscr{C}_{a}$ commutes with the prime operation, we immediately see that we also have $\mathscr{C}_{a} \iota^{B}=0$ for a type D spacetime, and that, conversely, if $\mathscr{C}_{a} O^{B}=$ $0=\mathscr{C}_{a} \iota^{B}$, then the spacetime is type D.

Note that, since $\mathscr{C}_{a}$ does not commute with complex conjugation, the condition $\mathscr{C}_{a} O^{B}=$ 0 does not imply $\mathscr{C}_{a} \bar{o}^{B^{\prime}}=0$; in fact we generally have $\mathscr{C}_{a} \bar{o}^{B^{\prime}} \neq 0$.

The result of Lemma 2.8 then explains the modified Killing spinor equation (1.7). For, in a type $\mathrm{D}$ spacetime, by Remark 2.2 we can write the 1 -form $f_{a}$ as in (1.9), and recalling the definition of the Teukolsky derivative given in the introduction (using now the more general form (2.38) for $B_{a}$ ), after an easy calculation we get

$$
\begin{aligned}
& \mathscr{C}_{A^{\prime}}{ }^{(A} O^{B)}=\Psi_{2}^{-w_{0} / 3} D_{A^{\prime}}\left(A\left[\Psi_{2}^{w_{0} / 3} o^{B)}\right],\right. \\
& \mathscr{C}_{A^{\prime}}\left(\iota^{B} \iota^{B)}=\Psi_{2}^{-w_{1} / 3} D_{A^{\prime}}\left(A\left[\Psi_{2}^{w_{1} / 3} \iota^{B)}\right] .\right.\right.
\end{aligned}
$$

Choosing $w_{0}=0, w_{1}=-1$, we immediately obtain (1.7) on a type D spacetime, as a consequence of Lemma 2.8. Note that, in particular, the vanishing of $\mathscr{C}_{a}\left[o^{B} \iota^{C}\right]$ gives the ordinary Killing spinor of type D spaces mentioned in the introduction. 


\subsection{Weighted local twistors}

A useful framework for dealing with conformal geometry in arbitrary dimensions is the tractor formalism, see e.g. [5]. When specialized to conformal spinor geometry in 4 dimensions, the tractor calculus becomes the local twistor formalism, which is a possible generalization of the original twistor theory to curved spacetimes, see [17, 6] and [15, section 6.9]. In this section we show that the distinguished Weyl connection of conformal type D spaces leads to a natural injection of spinor space into the local twistor bundle, and make some general remarks about the construction.

A local twistor can be represented as a pair of spinors $\left(\omega^{A}, \pi_{A^{\prime}}\right)$ such that under a conformal transformation, the local twistor itself is invariant, but its representation into spinor parts is changed according to $\widehat{\omega}^{A}=\omega^{A}, \widehat{\pi}_{A^{\prime}}=\pi_{A^{\prime}}+i \Upsilon_{A A^{\prime}} \omega^{A}$. This is usually described by the short exact sequence

$$
0 \rightarrow \mathbb{S}_{A^{\prime}} \rightarrow \mathbb{T}^{\alpha} \rightarrow \mathbb{S}^{A} \rightarrow 0
$$

where $\mathbb{T}^{\alpha}$ is the space of local twistors 7 , and the second and third maps are given respectively by $\pi_{A^{\prime}} \mapsto\left(0, \pi_{A^{\prime}}\right)$ and $\left(\omega^{A}, \pi_{A^{\prime}}\right) \mapsto \omega^{A}$. The sequence is then conformally invariant. The space $\mathbb{T}^{\alpha}$ is a vector bundle over $\mathscr{M}$ with structure group $\mathrm{SU}(2,2)$, and with a conformally invariant connection called local twistor transport, given by

$$
{ }^{\mathrm{T}} \nabla_{a} \mathrm{Z}^{\beta}:=\left(\nabla_{a} \omega^{B}+i \epsilon_{A}{ }^{B} \pi_{A^{\prime}}, \nabla_{a} \pi_{B^{\prime}}+i P_{A A^{\prime} C B^{\prime}} \omega^{C}\right),
$$

where $P_{A A^{\prime} B B^{\prime}}$ is the Schouten tensor (2.9). A global twistor is one which is parallel under local twistor transport, and in that case it coincides with the usual twistor concept in a (conformally) flat spacetime. The primary spinor part (i.e. the spinor part with all its indices at the upper position) of a global twistor is a Killing spinor: $\nabla_{A^{\prime} A} \omega^{B}=-i \epsilon_{A}{ }^{B} \pi_{A^{\prime}}$.

An exact sequence like (2.56) but with the arrows in the opposite direction can be obtained by means of the 1 -form (2.19). This is because (2.19) allows a natural injection of $\mathbb{S}^{A}$ into local twistor space, by mapping a spinor field $\omega^{A}$ (with conformal weight zero) to $\left(\omega^{A}, \alpha_{A^{\prime}}\right)$, where $\alpha_{A^{\prime}}=-i f_{A A^{\prime}} \omega^{A}$. In other words, we have the short exact sequence

$$
0 \rightarrow \mathbb{S}^{A} \rightarrow \mathbb{T}^{\alpha} \rightarrow \mathbb{S}_{A^{\prime}} \rightarrow 0
$$

where the second and third maps are now $\omega^{A} \mapsto\left(\omega^{A}, \alpha_{A^{\prime}}\right)$ and $\left(\omega^{A}, \pi_{A^{\prime}}\right) \mapsto \pi_{A^{\prime}}+i f_{A A^{\prime}} \omega^{A}$. The exactness of the sequence and its conformal invariance are easily checked. Consider for example a spin frame $\left\{o^{A}, \iota^{A}\right\}$, and choose the conformal weights $w_{0}=0$ and $w_{1}=-1$ in (2.1). Via (2.58), we have the local twistor $\mathrm{X}^{\alpha}=\left(o^{A}, \alpha_{A^{\prime}}\right)$. On the other hand, the pair $\mathrm{Y}^{\alpha}=\left(\iota^{A}, \beta_{A^{\prime}}\right)$, where $\beta_{A^{\prime}}:=-i f_{A A^{\prime}} \iota^{A}$, is a conformally weighted local twistor, with conformal weight $w=-1$, i.e. a section of $\mathbb{T}^{\alpha} \otimes \mathcal{E}[-1]$. But note that $\mathbf{X}^{\alpha}$ and $Y^{\alpha}$ are also GHP weighted: they have GHP types $\{1,0\}$ and $\{-1,0\}$ respectively. This situation then leads us naturally to the consideration of weighted local twistors.

We will say that $Z^{\alpha}$ is a weighted local twistor if, under conformal and GHP transformations, it transforms as $Z^{\alpha} \rightarrow \Omega^{w} Z^{\alpha}$ and $Z^{\alpha} \rightarrow \lambda^{p} \bar{\lambda}^{q} Z^{\alpha}$ respectively. In the spinor representation $\mathbf{Z}^{\alpha}=\left(\omega^{A}, \pi_{A^{\prime}}\right)$, both spinor parts have GHP type $\{p, q\}$, and their conformal behavior is $\widehat{\omega}^{A}=\Omega^{w} \omega^{A}, \widehat{\pi}_{A^{\prime}}=\Omega^{w}\left(\pi_{A^{\prime}}+i \Upsilon_{A A^{\prime}} \omega^{A}\right)$. The set of weighted local

\footnotetext{
${ }^{7}$ Greek letters $\alpha, \beta, \gamma, \ldots$ denote twistor indices, and take values in $\{0,1,2,3\}$.
} 
twistors can then be identified with the vector bundle $\mathbb{T}_{\{p, q\}}^{\alpha}[w]:=\mathbb{T}^{\alpha} \otimes \mathbb{E}_{\{p, q\}}[w]$. We can construct a connection on this structure by combining the usual local twistor transport (2.57) with the covariant derivative (2.37), extended to act 'trivially' on spinor indices. More precisely, we define

$$
{ }^{\mathrm{T}} \mathscr{C}_{a} Z^{\beta}:=\left(\oplus_{a} \omega^{B}+i \epsilon_{A}^{B} \pi_{A^{\prime}}, \oplus_{a} \pi_{B^{\prime}}+i P_{A A^{\prime} C B^{\prime}} \omega^{C}\right) .
$$

In this expression, we have $8 \oplus_{a} \omega^{B}=\nabla_{a} \omega^{B}+w f_{a} \omega^{B}+p\left(\omega_{a}+B_{a}\right) \omega^{B}+q\left(\bar{\omega}_{a}+C_{a}\right) \omega^{B}$, and analogously for $\oplus_{a} \pi_{B^{\prime}}$. One can then check that ${ }^{\mathrm{T}} \mathscr{C}_{a} Z^{\beta}$ is again a weighted local twistor, with conformal weight $w$ and GHP type $\{p, q\}$; therefore (2.59) gives a connection on $\mathbb{T}_{\{p, q\}}^{\alpha}[w]$. In this framework we can define a weighted global twistor as one which is parallel under weighted local twistor transport (2.59). This leads us to the definition of a weighted Killing spinor as the primary spinor part of a parallel weighted global twistor:

$$
\oplus_{A^{\prime} A^{\prime}} \omega^{B}=-i \epsilon_{A}^{B} \pi_{A^{\prime}}
$$

The generalization of this equation is

$$
\bigoplus_{A^{\prime}}\left(A \omega^{\left.B_{1} \ldots B_{n}\right)}=0\right.
$$

It is easily checked that $\bigoplus_{A^{\prime}}\left(A^{\left.B_{1} \ldots B_{n}\right)}=\mathscr{C}_{A^{\prime}}\left(A^{\left.A_{1} \ldots B_{n}\right)}\right.\right.$, therefore this definition coincides with (2.51) (the symmetrization being crucial for this to hold).

\section{Massless fields}

\subsection{Conformally covariant identities}

We now wish to find conformally covariant identities for massless free fields propagating in a background curved spacetime, and their possible relation to the identities (1.8).

Let $\varphi_{A_{1} \ldots A_{n}}$ be an arbitrary symmetric spinor field with conformal weight -1 and GHP type $\{0,0\}$. Recalling the definition of the conformally covariant derivative (2.15), for any 1 -form $f_{a}$ associated to a Weyl connection we have

$$
\nabla^{A_{1} A_{1}^{\prime}} \varphi_{A_{1} \ldots A_{n}}=\mathscr{C}^{A_{1} A_{1}^{\prime}} \varphi_{A_{1} \ldots A_{n}} .
$$

From now on we will use the 1 -form $f_{a}$ given by (2.19), since, as we have seen, it is naturally distinguished for the algebraically special spacetimes we are interested in, in relation to their almost-complex structure. The simplest operation that we can effect on $\mathscr{C}^{A_{1} A_{1}^{\prime}} \varphi_{A_{1} \ldots A_{n}}$ to get a conformally covariant second order differential operator, with $n$ totally symmetric unprimed indices, is to simply take an additional covariant derivative:

$$
\mathscr{C}_{A_{1}^{\prime}\left(A_{1}\right.} \mathscr{C}^{A_{1}^{\prime} B} \varphi_{\left.A_{2} \ldots A_{n}\right) B}
$$

It is tedious but straightforward to show that, in an arbitrary spacetime, we have

$$
\begin{aligned}
2 \mathscr{C}_{A_{1}^{\prime}\left(A_{1}\right.} \mathscr{C}^{A_{1}^{\prime} B} \varphi_{\left.A_{2} \ldots A_{n}\right) B}= & \left(\square_{\{-1 ; 0,0\}}-(n+2) \Psi_{2}+n \zeta\right) \varphi_{A_{1} \ldots A_{n}} \\
& -n \mu_{\left(A_{1}{ }^{B}\right.} \varphi_{\left.A_{2} \ldots A_{n}\right) B}-2(n-1) \Psi_{\left(A_{1} A_{2}\right.}{ }^{B C} \varphi_{\left.A_{3} \ldots A_{n}\right) B C},
\end{aligned}
$$

\footnotetext{
${ }^{8}$ the spinor $\omega^{A}$ and the GHP connection form $\omega_{a}$ should not be confused in this formula.
} 
where we have defined the conformally and GHP covariant wave operator

$$
\square_{\{w ; p, q\}}:=g^{a b} \mathscr{C}_{a} \mathscr{C}_{b}
$$

acting on $\mathbb{S}_{\{p, q\} A_{1} \ldots A_{n}}[w]$, and also $\zeta:=\sigma \sigma^{\prime}-\kappa \kappa^{\prime}, \mu_{A B}:=\chi_{\iota_{A} \iota_{B}}-\chi^{\prime} o_{A} O_{B}$, and $\chi:=$ $\left(\mathrm{p}^{\prime}+2 \rho^{\prime}-\bar{\rho}^{\prime}\right) \kappa-\left(\chi^{\prime}+2 \tau^{\prime}-\bar{\tau}\right) \sigma+2 \Psi_{1}$. We want to obtain equations for the components of $\varphi_{A_{1} \ldots A_{n}}$ in an arbitrary spin dyad $\left\{o^{A}, \iota^{A}\right\}$, where we choose the conformal weights $w_{0}=0$ and $w_{1}=-1$ (with respect to (2.1) ). The components are defined by

$$
\varphi_{k}:=\varphi_{A_{1} \ldots A_{k} A_{k+1} \ldots A_{n}} \iota^{A_{1}} \ldots \iota^{A_{k}} O^{A_{k+1} \ldots O^{A_{n}}} .
$$

The conformal weight of $\varphi_{k}$ is $w=-1-k$, and its GHP type is $\{n-2 k, 0\}$. Consider the extreme spin weight case; in particular the component $\varphi_{0}$. Defining $o^{A_{1} \ldots A_{n}}=o^{A_{1}} \ldots o^{A_{n}}$ and projecting on $o^{A_{1} \ldots A_{n}}$, after some tedious calculations (using (2.53)) we find

$$
2 o^{A_{1} \ldots A_{n}} \mathscr{C}_{A_{1}^{\prime}\left(A_{1}\right.} \mathscr{C}^{A_{1}^{\prime} B} \varphi_{\left.A_{2} \ldots A_{n}\right) B}=\left(\square_{\{-1 ; n, 0\}}-3 n \Psi_{2}\right) \varphi_{0}+F_{n}\left[\varphi_{1}\right]+G_{n}\left[\varphi_{2}\right],
$$

where $F_{n}\left[\varphi_{1}\right]=-2 n\left(-\kappa \mathrm{p}_{\mathscr{C}}^{\prime}+\sigma{\partial_{\mathscr{C}}^{\prime}}^{\prime}-\chi-\Psi_{1}\right) \varphi_{1}-4 \Psi_{1} \varphi_{1}$ and $G_{n}\left[\varphi_{2}\right]=-2(n-1) \Psi_{0} \varphi_{2}$. Imposing now the field equations $\mathscr{C}^{A_{1}^{\prime} A_{1}} \varphi_{A_{1} \ldots A_{n}}=0$, and assuming the components $\varphi_{1}$ and $\varphi_{2}$ to be arbitrary, we see that $\varphi_{0}$ satisfies a decoupled equation if and only if $\kappa=$ $\sigma=\Psi_{0}=\Psi_{1}=0$, that is, if and only if the spacetime is algebraically special, with $o^{A}$ aligned to the principal null direction. The decoupled equation in such case is

$$
\left(\square_{\{-1 ; n, 0\}}-3 n \Psi_{2}\right) \varphi_{0}=0 .
$$

Remark 3.1. Note that the condition $\kappa=\sigma=\Psi_{0}=\Psi_{1}=0$ imposed on the spacetime is well defined on the conformal class, since both the spin coefficients $\kappa, \sigma$ and the Weyl scalars are conformal densities, thus the condition extends to all metrics in the conformal class. This is just another way of saying that the algebraic speciality of a spacetime is common to the whole conformal class.

On the other hand, since the covariant derivative $\mathscr{C}_{a}$ commutes with the prime operation, from (3.6) we immediately deduce an analogous identity for the component with opposite extreme spin weight:

$$
2 \iota^{A_{1} \ldots A_{n}} \mathscr{C}_{A_{1}^{\prime}\left(A_{1}\right.} \mathscr{C}^{A_{1}^{\prime} B} \varphi_{\left.A_{2} \ldots A_{n}\right) B}=\left(\square_{\{-n-1 ;-n, 0\}}-3 n \Psi_{2}\right) \varphi_{n}+F_{n}^{\prime}\left[\varphi_{n-1}\right]+G_{n}^{\prime}\left[\varphi_{n-2}\right] .
$$

We then deduce that both extreme components, $\varphi_{0}$ and $\varphi_{n}$, satisfy decoupled equations if and only if it holds $\kappa=\sigma=\kappa^{\prime}=\sigma^{\prime}=\Psi_{0}=\Psi_{1}=\Psi_{3}=\Psi_{4}=0$, namely, if and only if the spacetime is of Petrov type D.

The previous discussion is valid for fields with arbitrary spin. We now particularize to the physically relevant cases of spin $1 / 2,1$ and 2 .

\subsubsection{Spin $\mathfrak{s}=1 / 2$}

The results just described apply directly. In particular, we have the following identities valid for an arbitrary spacetime:

$$
\begin{aligned}
& 2 o^{A} \mathscr{C}_{A^{\prime} A} \mathscr{C}^{A^{\prime} B} \varphi_{B}=\left(\square_{\{-1 ; 1,0\}}-3 \Psi_{2}\right) \varphi_{0}+F_{1}\left[\varphi_{1}\right], \\
& 2 \iota^{A} \mathscr{C}_{A^{\prime} A} \mathscr{C}^{A^{\prime} B} \varphi_{B}=\left(\square_{\{-2 ;-1,0\}}-3 \Psi_{2}\right) \varphi_{1}+F_{1}^{\prime}\left[\varphi_{0}\right] .
\end{aligned}
$$


The extreme component $\varphi_{0}=o^{A} \varphi_{A}$ of an arbitrary Weyl-Dirac field satisfies a decoupled wave-like equation if and only if the spacetime is algebraically special, with PND aligned to $o^{A}$. Both components $\varphi_{0}$ and $\varphi_{1}$ decouple if and only if the spacetime is of Petrov type D.

\subsubsection{Spin $\mathfrak{s}=1$}

For $\mathfrak{s}=1$ the previous results also apply directly. Besides the extreme weight cases, we have to consider the corresponding identity for spin weight zero; this can be calculated analogously to the others. For an arbitrary spacetime, we get the following identities:

$$
\begin{aligned}
& 2 o^{A B} \mathscr{C}_{A^{\prime}(A} \mathscr{C}^{A^{\prime} C} \varphi_{B) C}=\left(\square_{\{-1 ; 2,0\}}-6 \Psi_{2}\right) \varphi_{0}+F_{2}\left[\varphi_{1}\right]+G_{2}\left[\varphi_{2}\right], \\
& 2 o^{A} \iota^{B} \mathscr{C}_{A^{\prime}(A} \mathscr{C}^{A^{\prime} C} \varphi_{B) C}=\left(\square_{\{-2 ; 0,0\}}-2 \zeta\right) \varphi_{1}+H_{2}\left[\varphi_{2}\right]+H_{2}^{\prime}\left[\varphi_{0}\right], \\
& 2 \iota^{A B} \mathscr{C}_{A^{\prime}(A} \mathscr{C}^{A^{\prime} C} \varphi_{B) C}=\left(\square_{\{-3 ;-2,0\}}-6 \Psi_{2}\right) \varphi_{2}+F_{2}^{\prime}\left[\varphi_{1}\right]+G_{2}^{\prime}\left[\varphi_{0}\right],
\end{aligned}
$$

where we have defined $H_{2}\left[\varphi_{2}\right]:=2\left(\kappa \mathrm{b}_{\mathscr{C}}^{\prime}-\sigma \chi_{\mathscr{C}}^{\prime}+\chi-2 \Psi_{1}\right) \varphi_{2}$. As in the spin $\mathfrak{s}=1 / 2$ case, the extreme component of a generic Maxwell field decouples if and only if the spacetime is algebraically special. The spin weight zero component, on the other hand, decouples if and only if the spacetime is type D.

\subsubsection{Spin $\mathfrak{s}=2$}

This case is much more subtle than the previous ones, since if we intend to describe "massless free fields of spin 2 propagating in a curved background spacetime" as perturbations of the Weyl curvature spinor, then the conformally covariant description that we developed is not appropriate, since $\Psi_{A B C D}$ has conformal weight $w=0$, and it should have $w=-1$ in order for our general formulae to be valid. This suggests that we describe the required massless spin 2 fields in terms of a rescaled Weyl spinor, defined in the following way. Given the conformal class $\left[g_{a b}\right]$ associated to $g_{a b}$, for an arbitrary representative $\left[g_{a b}\right] \ni \widehat{g}_{a b}=\Omega^{2} g_{a b}$ we introduce

$$
\varphi_{A B C D}:=\Omega^{-1} \Psi_{A B C D}
$$

This object has conformal weight $w=-1$, and it is actually very used in studies of conformal and asymptotic aspects of the Einstein field equations and in the analysis of conformal infinity in General Relativity, see e.g. [10, [15, sections 9.6, 9.7] and [18, Ch. 8 and 10]. In fact, following closely Penrose \& Rindler [15], we can think of (3.14) as the "gravitational spin 2 field" (see discussion around eq. (9.6.40) and theorem (9.6.41) in [15]), in the sense that the property of peeling allows to interpret the components of (3.14) as describing the gravitational radiation field near conformal infinity in asymptotically simple spacetimes ([15, eq. (9.7.38)]).

Let $\left(\mathscr{M}, g_{a b}(\varepsilon)\right)$ be a monoparametric family of spacetimes, such that $g_{a b}(0)$ satisfies the vacuum Einstein equations with cosmological constant. Consider the conformal class associated to $g_{a b}(\varepsilon)$, denoted as $\left[g_{a b}(\varepsilon)\right]$, where $\widehat{g}_{a b}(\varepsilon) \in\left[g_{a b}(\varepsilon)\right]$ if and only if there exists $\Omega(\varepsilon)>0$ such that $\widehat{g}_{a b}(\varepsilon)=\Omega^{2}(\varepsilon) g_{a b}(\varepsilon)$. Note that we impose the conformal factor to depend on the parameter $\varepsilon$; in particular, we take $\Omega(0) \equiv \AA=$ constant (the reason for this can be inferred from the calculations given in appendix $\mathrm{A}$ ). For an arbitrary 
representative in $\left[g_{a b}(\varepsilon)\right]$, we have $\varphi_{A B C D}(\varepsilon)=\Omega^{-1}(\varepsilon) \Psi_{A B C D}(\varepsilon)$. Linearizing equation (3.6) for $n=4$, we find 9

$$
\left.\frac{d}{d \varepsilon}\right|_{\varepsilon=0}\left\{2 o^{A B C D} \mathscr{C}_{A^{\prime}(A} \mathscr{C}^{A^{\prime} E} \varphi_{B C D) E}\right\}=\left(\square_{\{-1 ; 4,0\}}-12 \Psi_{2}\right) \dot{\varphi}_{0}-6 \varphi_{2} \dot{\Psi}_{0}+\dot{B}_{0},
$$

where

$$
\dot{B}_{0}=\left(\dot{\square}_{\{-1 ; 4,0\}}-12 \dot{\Psi}_{2}\right) \varphi_{0}-6 \Psi_{0} \dot{\varphi}_{2}+\dot{F}_{4}
$$

Now, the field equations describing gravitational perturbations are the linearized vacuum (with cosmological constant) Einstein equations, which imply the linearized Bianchi identities $\left.\frac{d}{d \varepsilon}\right|_{\varepsilon=0}\left(\nabla^{A^{\prime} A} \Psi_{A B C D}\right)=0$. Replacing $\varphi_{A B C D}$ by $\Omega^{-1} \Psi_{A B C D}$ in the left hand side of (3.15) and imposing the just mentioned field equations, after some tedious calculations (see appendix A) we get that $\dot{\varphi}_{0}$ satisfies a decoupled equation if and only if $\kappa=\sigma=\Psi_{0}=\Psi_{1}=0$, that is, if and only if the background spacetime is algebraically special, with PND aligned to $o^{A}$. Note that in such case we have $\dot{\varphi}_{0}=\Omega^{-1} \dot{\Psi}_{0}$. For the component with opposite extreme spin weight, namely $\dot{\varphi}_{4}$, we have

$$
\left.\frac{d}{d \varepsilon}\right|_{\varepsilon=0}\left\{2 \iota^{A B C D} \mathscr{C}_{A^{\prime}(A} \mathscr{C}^{A^{\prime} E} \varphi_{B C D) E}\right\}=\left(\square_{\{-5 ;-4,0\}}-12 \Psi_{2}\right) \dot{\varphi}_{4}-6 \varphi_{2} \dot{\Psi}_{4}+\dot{B}_{4},
$$

where $\dot{B}_{4}=\left(\dot{B}_{0}\right)^{\prime}$. Imposing the field equations and using the explicit expression of $\dot{B}_{4}$, we then see that both extreme components, $\dot{\varphi}_{0}$ and $\dot{\varphi}_{4}$, decouple if and only if it holds $\kappa=\sigma=\kappa^{\prime}=\sigma^{\prime}=\Psi_{0}=\Psi_{1}=\Psi_{3}=\Psi_{4}=0$, i.e. if and only if the background spacetime is of Petrov type D. In that case we have $\dot{\varphi}_{0}=\stackrel{\Omega}{\Omega}^{-1} \dot{\Psi}_{0}$ and $\dot{\varphi}_{4}=\stackrel{\Omega}{\Omega}^{-1} \dot{\Psi}_{4}$.

Finally, consider the spin weight zero component $\varphi_{2}$. A calculation similar to (3.6) leads to the following result in an arbitrary spacetime:

$$
2 o^{A B}{ }_{\iota} C D \mathscr{C}_{A^{\prime}(A} \mathscr{C}^{A^{\prime} E} \varphi_{B C D) E}=\square_{\{-3 ; 0,0\}} \varphi_{2}+B_{2},
$$

where

$$
\begin{aligned}
B_{2}= & -\left(\kappa \mathrm{p}_{\mathscr{C}}^{\prime}-\sigma \partial_{\mathscr{C}}^{\prime}-4 \chi+16 \Psi_{1}\right) \varphi_{3}-\left(\kappa^{\prime} \mathrm{p}_{\mathscr{C}}-\sigma^{\prime} \partial_{\mathscr{C}}-4 \chi^{\prime}+16 \Psi_{3}\right) \varphi_{1} \\
& -8 \zeta \varphi_{2}-6\left(\Psi_{0} \varphi_{4}+\Psi_{4} \varphi_{0}\right) .
\end{aligned}
$$

Linearizing equation (3.18), the only interesting case we find is when the background spacetime is type $\mathrm{D}$, where it holds

$$
\left.\frac{d}{d \varepsilon}\right|_{\varepsilon=0}\left\{2 o^{A B} \iota^{C D} \mathscr{C}_{A^{\prime}(A} \mathscr{C}^{A^{\prime} E} \varphi_{B C D) E}\right\}=\square_{\{-3 ; 0,0\}} \dot{\varphi}_{2}+\dot{\square}_{\{-3 ; 0,0\}} \varphi_{2} .
$$

Now, it is important to note that, on shell, the left hand side of (3.20) does not vanish, but it is equal to $\square_{\{-3 ; 0,0\}}\left[\Psi_{2} \dot{\Omega}^{-1}\right]$, see appendix $\mathrm{A}$. We then see that, on shell, $\dot{\varphi}_{2}$ does not decouple, since the terms $\square_{\{-3 ; 0,0\}}\left[\Psi_{2} \dot{\Omega}^{-1}\right]$ and $\dot{\square}_{\{-3 ; 0,0\}} \varphi_{2}$ do not vanish. On the other hand, $\square_{\{-3 ; 0,0\}}\left[\Psi_{2} \dot{\Omega}^{-1}\right]$ cancels one of the terms appearing in the right hand side of (3.20) (after replacing $\dot{\varphi}_{2}=\Psi_{2} \dot{\Omega}^{-1}+\stackrel{\Omega}{\Omega}^{-1} \dot{\Psi}_{2}$ ), so that, on shell, we are left with the equation

$$
\square_{\{-3 ; 0,0\}}\left[\AA^{-1} \dot{\Psi}_{2}\right]+\dot{\square}_{\{-3 ; 0,0\}} \varphi_{2}=0 .
$$

\footnotetext{
${ }^{9}$ linearized quantities are denoted with a dot, e.g. $\dot{T}:=\left.\frac{d}{d \varepsilon}\right|_{\varepsilon=0}[T(\varepsilon)]$; quantities without a dot in the right hand side of (3.15) are understood as evaluated in the background.
} 
Summarizing, the conformally covariant identities we find for the case in which the background spacetime is type $\mathrm{D}$ are:

$$
\begin{aligned}
\left.\frac{d}{d \varepsilon}\right|_{\varepsilon=0}\left\{2 o^{A B C D} \mathscr{C}_{A^{\prime}(A} \mathscr{C}^{A^{\prime} E} \varphi_{B C D) E}\right\} & =\left(\square_{\{-1 ; 4,0\}}-18 \Psi_{2}\right) \dot{\varphi}_{0}, \\
\left.\frac{d}{d \varepsilon}\right|_{\varepsilon=0}\left\{2 o^{A B} \iota^{C D} \mathscr{C}_{A^{\prime}(A} \mathscr{C}^{A^{\prime} E} \varphi_{B C D) E}\right\} & =\square_{\{-3 ; 0,0\}} \dot{\varphi}_{2}+\dot{\square}_{\{-3 ; 0,0\}} \varphi_{2}, \\
\left.\frac{d}{d \varepsilon}\right|_{\varepsilon=0}\left\{2 \iota^{A B C D} \mathscr{C}_{A^{\prime}(A} \mathscr{C}^{A^{\prime} E} \varphi_{B C D) E}\right\} & =\left(\square_{\{-5 ;-4,0\}}-18 \Psi_{2}\right) \dot{\varphi}_{4} .
\end{aligned}
$$

The left hand sides of equations (3.22) and (3.24) vanish on shell, leaving us with decoupled equations for $\dot{\varphi}_{0}$ and $\dot{\varphi}_{4}$ respectively, where the normally hyperbolic operator $\square_{\{w ; p, q\}}$ (defined in (3.4) ) has a well defined geometrical meaning in terms of conformal and GHP covariance (see section 2.4). The left hand side of (3.23) does not vanish on shell, and the discussion between equations (3.20) and (3.21) applies.

\subsection{Relation with Teukolsky operators}

In the previous subsection we found conformally covariant identities for fields with spin 1/2, 1 and 2, and we also found the conditions that must satisfy the metrics of the conformal class associated to the background spacetimes in order for the field components to satisfy wave-like decoupled equations. In order to relate these results with the main identity (1.8), we need the relation between the conformally covariant operators that we used, and the Teukolsky operators that appear in (1.8). We have:

Lemma 3.2. Consider an Einstein spacetime of Petrov type D, and let $\eta$ be a conformal scalar density of type $\{w ; p, 0\}$. The relation between the conformally-GHP covariant wave operator $\square_{\{w ; p, 0\}}$ and the Teukolsky operator $\Phi_{p}$ is given by:

$$
\square_{\{w ; p, 0\}} \eta=\Psi_{2}^{-(w+1) / 3}\left(\square_{p}+2 \Psi_{2}+\frac{R}{6}\right)\left(\Psi_{2}^{(w+1) / 3} \eta\right) .
$$

Proof. Recall the definition of the Teukolsky derivative (acting on GHP type $\{p, 0\}$ quantities), $D_{a}=\Theta_{a}+p B_{a}$ (where $B_{a}$ is given by (2.38)), and the associated wave operator $\square_{p}=D^{a} D_{a}$. Since the spacetime is type D we can express the 1 -form (2.19) as (1.9) $)^{10}$, then it is easy to show that, for an arbitrary number $z \in \mathbb{R}$, we have

$$
\Psi_{2}^{-z / 3} \square_{p}\left(\Psi_{2}^{z / 3} \eta\right)=\Phi_{p} \eta+2 z f^{a} D_{a} \eta+z\left[\nabla_{a} f^{a}+z f_{a} f^{a}\right] \eta
$$

On the other hand, for $\square_{\{w ; p, 0\}} \eta$ we find

$$
\square_{\{w ; p, 0\}} \eta=\square_{p} \eta+2(w+1) f^{a} D_{a} \eta+w\left[\nabla_{a} f^{a}+(w+2) f_{a} f^{a}\right] \eta .
$$

Using now (3.26) with $z=w+1$ and the identity $\nabla_{a} f^{a}+f_{a} f^{a}=-\left(2 \Psi_{2}+\frac{R}{6}\right)$, the result follows.

Using Lemma 3.2, we deduce immediately the following identities valid for perturbations of an Einstein type D spacetime (where $\lambda$ is the cosmological constant):

\footnotetext{
${ }^{10}$ Recall that this way one is breaking the explicit conformal covariance of the formalism, see Remark 2.2
} 
Spin $\mathfrak{s}=1 / 2$ :

$$
\begin{aligned}
2 o^{B} \mathscr{C}_{A^{\prime} B} \mathscr{C}^{A^{\prime} A} \varphi_{A} & =\left(\Phi_{+1}-\Psi_{2}+\frac{2}{3} \lambda\right) \varphi_{0}, \\
2 \iota^{B} \mathscr{C}_{A^{\prime} B} \mathscr{C}^{A^{\prime} A} \varphi_{A} & =\Psi_{2}^{1 / 3}\left(\Phi_{-1}-\Psi_{2}+\frac{2}{3} \lambda\right)\left[\Psi_{2}^{-1 / 3} \varphi_{1}\right] .
\end{aligned}
$$

Spin $\mathfrak{s}=1$ :

$$
\begin{aligned}
2 o^{A B} \mathscr{C}_{A^{\prime}(A} \mathscr{C}^{A^{\prime} C} \varphi_{B) C} & =\left(\Phi_{+2}-4 \Psi_{2}+\frac{2}{3} \lambda\right) \varphi_{0}, \\
2 o^{A} \iota^{B} \mathscr{C}_{A^{\prime}(A} \mathscr{C}^{A^{\prime} C} \varphi_{B) C} & =\Psi_{2}^{1 / 3}\left(\square+2 \Psi_{2}+\frac{2}{3} \lambda\right)\left[\Psi_{2}^{-1 / 3} \varphi_{1}\right], \\
2 \iota^{A B} \mathscr{C}_{A^{\prime}(A} \mathscr{C}^{A^{\prime} C} \varphi_{B) C} & =\Psi_{2}^{2 / 3}\left(\square_{-2}-4 \Psi_{2}+\frac{2}{3} \lambda\right)\left[\Psi_{2}^{-2 / 3} \varphi_{2}\right] .
\end{aligned}
$$

Spin $\mathfrak{s}=2$ :

$$
\begin{aligned}
\left.\frac{d}{d \varepsilon}\right|_{\varepsilon=0}\left\{2 o^{A B C D} \mathscr{C}_{A^{\prime}(A} \mathscr{C}^{A^{\prime} E} \varphi_{B C D) E}\right\} & =\left(\Phi_{+4}-16 \Psi_{2}+\frac{2}{3} \lambda\right) \dot{\varphi}_{0}, \\
\left.\frac{d}{d \varepsilon}\right|_{\varepsilon=0}\left\{2 o^{A B} \iota^{C D} \mathscr{C}_{A^{\prime}(A} \mathscr{C}^{A^{\prime} E} \varphi_{B C D) E}\right\} & =\Psi_{2}^{2 / 3}\left(\square+2 \Psi_{2}+\frac{2}{3} \lambda\right)\left[\Psi_{2}^{-2 / 3} \dot{\varphi}_{2}\right]+\dot{\square}_{\{-3 ; 0,0\}} \varphi_{2}, \\
\left.\frac{d}{d \varepsilon}\right|_{\varepsilon=0}\left\{2 \iota^{A B C D} \mathscr{C}_{A^{\prime}(A} \mathscr{C}^{A^{\prime} E} \varphi_{B C D) E}\right\} & =\Psi_{2}^{4 / 3}\left(\Phi_{-4}-16 \Psi_{2}+\frac{2}{3} \lambda\right)\left[\Psi_{2}^{-4 / 3} \dot{\varphi}_{4}\right] .
\end{aligned}
$$

Finally, it is straightforward to show that

$$
\mathscr{C}_{A_{1}^{\prime}\left(A_{1}\right.} \mathscr{C}^{A_{1}^{\prime} B} \varphi_{\left.A_{2} \ldots A_{n}\right) B}=\left(\nabla_{A_{1}^{\prime}\left(A_{1}\right.}-n f_{A_{1}^{\prime}\left(A_{1}\right.}\right) \nabla^{A_{1}^{\prime} B} \varphi_{\left.A_{2} \ldots A_{n}\right) B},
$$

thus we recover the main identity (1.8) (recall that by Remark 2.2 we have $f_{a}=A_{a}$ in type D). Note that the formulation of section 3.1 is actually more general since it deals with all algebraically special spacetimes, not just the type D.

\subsection{The conformally covariant Laplace-de Rham operator}

In the case of integer spin, the spinorial operator (3.2) admits a description in tensor terms. Since in curved spacetimes the only interesting cases of integer spin fields correspond to spin 1 and 2, we now briefly discuss the tensorial structure of the operator in these cases.

For 4-dimensional spacetimes, it was found in [3] that the tensor structure of the spinorial operator on the right hand side of (3.36) is that of a "modified" Laplace-de Rham operator acting on tensor valued differential forms. The same idea applies here, with the only difference that now the operator adopts a more 'symmetric' form in terms of a covariant exterior derivative associated to $\mathscr{C}_{a}$. More precisely, for a tensor valued differential form (with well defined conformal weight) $\omega_{a_{1} \ldots a_{k} b_{1} \ldots b_{l}}=\omega_{a_{1} \ldots a_{k}\left[b_{1} \ldots b_{l}\right]}$, we define the covariant exterior derivative $\mathcal{D}_{\mathscr{C}}$ and its adjoint $\mathcal{D}_{\mathscr{C}}^{\dagger}$ by

$$
\begin{aligned}
&\left(\mathcal{D}_{\mathscr{C}} \omega\right)_{a_{1} \ldots a_{k} b_{1} \ldots b_{l+1}}:=(l+1) \mathscr{C}_{\left[b_{1}\right.} \omega_{\left.\left|a_{1} \ldots a_{k}\right| b_{2} \ldots b_{l+1}\right]}, \\
&\left(\mathcal{D}_{\mathscr{C}}^{\dagger} \omega\right)_{a_{1} \ldots a_{k} b_{1} \ldots b_{l-1}}:=-\mathscr{C}^{c} \omega_{a_{1} \ldots a_{k} c b_{1} \ldots b_{l-1}} .
\end{aligned}
$$

Let $F_{a b}$ and $K_{a b c d}$ be the tensorial analogues of the totally symmetric spinors $\varphi_{A B}$ and $\varphi_{A B C D}$, i.e.

$$
\begin{aligned}
& F_{a b}=\varphi_{A B} \bar{\epsilon}_{A^{\prime} B^{\prime}}+\bar{\varphi}_{A^{\prime} B^{\prime}} \epsilon_{A B}, \\
& K_{a b c d}=\varphi_{A B C D} \bar{\epsilon}_{A^{\prime} B^{\prime}} \bar{\epsilon}_{C^{\prime} D^{\prime}}+\bar{\varphi}_{A^{\prime} B^{\prime} C^{\prime} D^{\prime}} \epsilon_{A B} \epsilon_{C D}
\end{aligned}
$$

(where we are considering $K_{a b c d}=K_{a b[c d]}$ as a tensor valued 2-form with the extra tensorial indices $a b) . F_{a b}$ has conformal weight $w=0$, and $K_{a b c d}$ has $w=1$. Then: 
Lemma 3.3. With the definitions (3.37) and (3.38), we have the following identities:

$$
\begin{aligned}
& -\frac{1}{2}\left[\left(\mathcal{D}_{\mathscr{C}} \mathcal{D}_{\mathscr{C}}^{\dagger}+\mathcal{D}_{\mathscr{C}}^{\dagger} \mathcal{D}_{\mathscr{C}}\right) F\right]_{a b}=\mathscr{C}_{E^{\prime}(A} \mathscr{C}^{E^{\prime} E} \varphi_{B) E} \bar{\epsilon}_{A^{\prime} B^{\prime}}+\mathscr{C}_{E\left(A^{\prime}\right.} \mathscr{C}^{E E^{\prime}} \bar{\varphi}_{\left.B^{\prime}\right) E^{\prime}} \epsilon_{A B}, \\
& -\frac{1}{2}\left[\left(\mathcal{D}_{\mathscr{C}} \mathcal{D}_{\mathscr{C}}^{\dagger}+\mathcal{D}_{\mathscr{C}}^{\dagger} \mathcal{D}_{\mathscr{C}}\right) K\right]_{a b c d}=\mathscr{C}_{E^{\prime}(C} \mathscr{C}^{E^{\prime} E} \varphi_{|A B E| D)} \bar{\epsilon}_{A^{\prime} B^{\prime}} \bar{\epsilon}_{C^{\prime} D^{\prime}} \\
& +\mathscr{C}_{E\left(C^{\prime}\right.} \mathscr{C}^{E E^{\prime}} \bar{\varphi}_{\left.\left|A^{\prime} B^{\prime} E^{\prime}\right| D^{\prime}\right)} \epsilon_{A B} \epsilon_{C D} .
\end{aligned}
$$

The 4-dimensional "spin $\mathfrak{s}$ modified" Laplace-de Rham operator defined in [3] is then the conformally covariant Laplace-de Rham operator

$$
\mathcal{D}_{\mathscr{C}} \mathcal{D}_{\mathscr{C}}^{\dagger}+\mathcal{D}_{\mathscr{C}}^{\dagger} \mathcal{D}_{\mathscr{C}}
$$

acting on tensor valued differential forms with well defined conformal weight.

\section{Conclusions}

In this work we have developed a conformally and GHP covariant formalism for dealing with the massless free field equations (1.1) in (4-dimensional) algebraically special Einstein spacetimes, and we have shown that the operators associated to well known equations in the literature find a natural geometrical interpretation in this framework. The main tool of the construction is the almost-complex structure (2.18) and its unique associated Weyl connection (2.17) for the conformal manifold. Algebraically special spacetimes have preferred null directions on the geometry, and after adapting a null frame and its 'gauge symmetry' to them we showed that the covariant derivative naturally induced (from the conformal structure) in associated vector bundles is precisely the Teukolsky connection, see Theorem 2.6. Furthermore, the almost-complex structure is integrable in type D spaces and then they become complex Hermitian manifolds.

A natural interpretation of the 'ordinary' and 'generalized' hidden symmetries (given respectively by (1.3) and (1.7)) persistent on black hole perturbations has also emerged from our formalism, since, on the one hand, by Lemma 2.3 the existence of a conformal Killing-Yano tensor (or its associated Killing spinor) in type D spacetimes can be thought to be a consequence of the presence of a Kähler metric in the conformal Hermitian class, and more generally, the Killing spinor equations are just the reflection of the type D principal spinors being parallel with respect to the natural Weyl-GHP connection, which in turn (by Lemma 2.8) is a consequence of algebraic speciality. In this sense the spin lowering/raising mechanism derivable from the identities given in section 3.1 is closer in spirit to the original (and simpler) Penrose's work [16], which uses covariantly constant spinors in Minkowski.

On the other hand, conformal spinor geometry is particularly well suited for the local twistor formalism, and we have shown that the preferred Weyl connection leads to a natural local twistor exact sequence (2.58) in the reversed order to the standard one, which results in the construction of weighted local twistors. We introduced a connection on the weighted twistor bundle, and showed that the 'weighted Killing spinor notion' deduced from it coincides with our earlier definition.

Finally, we mention that the generalized Teukolsky connection and the closely related 'weighted Killing fields' found in [3] for perturbations of higher dimensional spacetimes, can be shown to follow the same principle as the one exploited here, namely conformal 
and GHP covariance. However, the question about conformal invariance of field equations in higher dimensions is much more subtle than in the 4-dimensional case (in particular, Maxwell fields in $d \neq 4$ are not conformally invariant).

\section{Acknowledgements}

I would like to thank Gustavo Dotti, Martin Reiris and Oscar Reula for very helpful discussions. This work is supported by CONICET (Argentina).

\section{A Some details of calculations for spin 2}

In this appendix we give some details of the calculations performed in section 3.1 .3 corresponding to spin $\mathfrak{s}=2$. We will use the notation and conventions of that section.

We are studying equations for the spin 2 field (3.14) in the context of gravitational perturbations. The field equations in this case are not $\nabla^{A^{\prime} A} \varphi_{A B C D}=0$, but the linearized Bianchi identities $\left.\frac{d}{d \varepsilon}\right|_{\varepsilon=0}\left(\nabla^{A^{\prime} A} \Psi_{A B C D}\right)=0$. Replacing $\varphi_{A B C D}=\Omega^{-1} \Psi_{A B C D}$, we have:

$$
\begin{aligned}
\mathscr{C}_{A^{\prime} A} \mathscr{C}^{A^{\prime} E} \varphi_{B C D E}= & \Omega^{-1}\left(\nabla_{A^{\prime} A}-4 f_{A^{\prime} A}\right) \nabla^{A^{\prime} E} \Psi_{B C D E}+\Psi_{B C D E}\left(\nabla_{A^{\prime} A}-4 f_{A^{\prime} A}\right) \nabla^{A^{\prime} E} \Omega^{-1} \\
& +\left(\nabla^{A^{\prime} E} \Omega^{-1}\right)\left(\nabla_{A^{\prime} A} \Psi_{B C D E}\right)+\left(\nabla_{A^{\prime} A} \Omega^{-1}\right)\left(\nabla^{A^{\prime} E} \Psi_{B C D E}\right) .
\end{aligned}
$$

Now let $\lambda^{A B C D}$ be some of the spinors in the set $\left\{O^{A B C D}, O^{(A} O^{B} \iota^{C} \iota^{D)}, \iota^{A B C D}\right\}$. Projecting (A.1) over $\lambda^{A B C D}$, linearizing, and taking into account the background assumptions $\left.\left(\nabla^{A^{\prime} A} \Psi_{A B C D}\right)\right|_{\varepsilon=0}=0$ and $\left.\left(\nabla_{a} \Omega\right)\right|_{\varepsilon=0}=0$ (since $\Omega(0)=\stackrel{\Omega}{\Omega}=$ const.), we get

$$
\begin{aligned}
\left.\frac{d}{d \varepsilon}\right|_{\varepsilon=0}\left\{\lambda^{A B C D} \mathscr{C}_{A^{\prime} A} \mathscr{C}^{A^{\prime} E} \varphi_{B C D E}\right\}= & \left.\stackrel{\Omega}{ }^{-1} \frac{d}{d \varepsilon}\right|_{\varepsilon=0}\left\{\lambda^{A B C D}\left(\nabla_{A^{\prime} A}-4 f_{A^{\prime} A}\right) \nabla^{A^{\prime} E} \Psi_{B C D E}\right\} \\
& +\left.\frac{d}{d \varepsilon}\right|_{\varepsilon=0}\left\{\lambda^{A B C D} \Psi_{B C D E}\left(\nabla_{A^{\prime} A}-4 f_{A^{\prime} A}\right) \nabla^{A^{\prime} E} \Omega^{-1}\right\} \\
& +\left.\frac{d}{d \varepsilon}\right|_{\varepsilon=0}\left\{\lambda^{A B C D}\left(\nabla^{A^{\prime} E} \Omega^{-1}\right)\left(\nabla_{A^{\prime} A} \Psi_{B C D E}\right)\right\} .
\end{aligned}
$$

For $\lambda^{A B C D}=o^{A B C D}$, it is straightforward to see that, if the background spacetime is algebraically special (so that $\kappa(0)=\sigma(0)=\Psi_{0}(0)=\Psi_{1}(0)=0$ ) the second and third lines in the right hand side of (A.2) vanish, thus the left hand side of the identity (3.15) vanishes on shell and we get the result mentioned in that section.

For $\lambda^{A B C D}=o^{(A} O^{B} \iota^{C} \iota^{D)}$, a tedious calculation assuming that the background spacetime is type $\mathrm{D}$ shows that (A.2) becomes

$$
\begin{aligned}
\left.\frac{d}{d \varepsilon}\right|_{\varepsilon=0}\left\{o^{(A} O^{B} \iota^{C} \iota^{D)} \mathscr{C}_{A^{\prime} A} \mathscr{C}^{A^{\prime} E} \varphi_{B C D E}\right\}= & \left.\stackrel{\Omega}{ }^{-1} \frac{d}{d \varepsilon}\right|_{\varepsilon=0}\left\{o^{(A} o^{B} \iota^{C} \iota^{D)}\left(\nabla_{A^{\prime} A}-4 f_{A^{\prime} A}\right) \nabla^{A^{\prime} E} \Psi_{B C D E}\right\} \\
& +\frac{1}{2} \Psi_{2}\left(\square+2 f^{a} \nabla_{a}\right) \dot{\Omega}^{-1} .
\end{aligned}
$$

Now, using the definition of the conformal wave operator $\square_{\{w ; p, q\}}$ and some identities of the background (type D) spacetime, it is not difficult to show that

$$
\Psi_{2}\left(\square+2 f^{a} \nabla_{a}\right) \dot{\Omega}^{-1}=\square_{\{-3 ; 0,0\}}\left[\Psi_{2} \dot{\Omega}^{-1}\right],
$$

then the discussion between equations (3.20) and (3.21) follows. 


\section{References}

[1] S. Aksteiner and L. Andersson, Linearized gravity and gauge conditions, Class. Quant. Grav. 28, 065001 (2011) arXiv:1009.5647 [gr-ac]].

[2] B. Araneda, Symmetry operators and decoupled equations for linear fields on black hole spacetimes, Class. Quant. Grav. 34, no. 3, 035002 (2017) arXiv:1610.00736 [gr-qc]].

[3] B. Araneda, Generalized wave operators, weighted Killing fields, and perturbations of higher dimensional spacetimes, Class. Quant. Grav. 35, no. 7, 075015 (2018) arXiv:1711.09872 [gr-qc]].

[4] T. N. Bailey, Complexified conformal almost-Hermitian structures and the conformally invariant eth and thorn operators, Class. Quant. Grav. 8, no. 1, (1991)

[5] S. Curry and A. R. Gover, An introduction to conformal geometry and tractor calculus, with a view to applications in general relativity, [arXiv:1412.7559 [math.DG]].

[6] K. Dighton, An introduction to the theory of local twistors, Int. J. Theor. Phys., vol. 11, no 1, p. 31-43 (1974)

[7] E. J. Flaherty Jr., An integrable structure for type D spacetimes, Phys. Lett. A 46 , 391-392 (1974)

[8] E. J. Flaherty Jr., Hermitian and Kählerian Geometry in Relativity, Springer Lecture Notes in Physics, Vol. 46 (Springer-Verlag, New York, 1976)

[9] H. Friedrich, Twistor connection and normal conformal Cartan connection, Gen. Relat. Gravit. (1977) 8: 303

[10] H. Friedrich, Smoothness at null infinity and the structure of initial data. In: Chruściel, P. T., and Friedrich, H. (eds), The Einstein Equations and the Large Scale Behavior of Gravitational Fields. 50 years of the Cauchy problem in general relativity, Birkhausser, 2004

[11] R. P. Geroch, A. Held and R. Penrose, A space-time calculus based on pairs of null directions, J. Math. Phys. 14, 874 (1973).

[12] A. R. Gover and P. Nurowski, Calculus and invariants on almost complex manifolds, including projective and conformal geometry, Illinois J. Math. 57 (2013), no. 2, 383427 arXiv:1208.0648 [math.DG]!.

[13] L. J. Mason, L. P. Hughston and P. Z. Kobak, Further advances in twistor theory. Vol. 2: Integrable systems, conformal geometry and gravitation, Harlow, UK: Longman (1995) 274 p. (Pitman research notes in mathematics series: 232)

[14] R. Penrose and W. Rindler, Spinors And Space-time. 1. Two Spinor Calculus And Relativistic Fields, Cambridge, Uk: Univ. Pr. ( 1984) 458 P. ( Cambridge Monographs On Mathematical Physics) 
[15] R. Penrose and W. Rindler, Spinors And Space-time. Vol. 2: Spinor And Twistor Methods In Space-time Geometry, Cambridge, Uk: Univ. Pr. ( 1986) 501p

[16] R. Penrose, Zero rest mass fields including gravitation: Asymptotic behavior, Proc. Roy. Soc. Lond. A 284 (1965) 159.

[17] R. Penrose and M. MacCallum, Twistor theory: an approach to the quantisation of fields and space-time, Physics Reports 6.4 (1973): 241-315.

[18] J. A. Valiente Kroon, Conformal Methods in General Relativity, Cambridge, UK: Cambridge University Press (2016). 